\title{
Precision Medicine in Control of Visceral Leishmaniasis Caused by L. donovani
}

\author{
Eduard E. Zijlstra* \\ Clinical Sciences, Rotterdam Centre for Tropical Medicine, Rotterdam, Netherlands
}

\section{OPEN ACCESS}

Edited by:

Om Prakash Singh,

Banaras Hindu University, India

Reviewed by:

Chiraniib Pal,

West Bengal State University, India

Manju Jain,

Central University of Punjab, India

Rajib Chowdhury,

National Institute of Preventive and

Social Medicine (NIPSOM),

Bangladesh

*Correspondence:

Eduard E. Zijlstra

e.e.zij/stra@roctm.com

Specialty section:

This article was submitted to

Parasite and Host,

a section of the journal

Frontiers in Cellular and

Infection Microbiology

Received: 10 May 2021 Accepted: 28 September 2021 Published: 09 November 2021

Citation:

Zijlstra EE (2021) Precision Medicine in

Control of Visceral Leishmaniasis

Caused by L. donovani.

Front. Cell. Infect. Microbiol. 11:707619.

doi: 10.3389/fcimb.2021.707619
Precision medicine and precision global health in visceral leishmaniasis $(\mathrm{VL})$ have not yet been described and could take into account how all known determinants improve diagnostics and treatment for the individual patient. Precision public health would lead to the right intervention in each VL endemic population for control, based on relevant population-based data, vector exposures, reservoirs, socio-economic factors and other determinants. In anthroponotic VL caused by $L$. donovani, precision may currently be targeted to the regional level in nosogeographic entities that are defined by the interplay of the circulating parasite, the reservoir and the sand fly vector. From this 5 major priorities arise: diagnosis, treatment, PKDL, asymptomatic infection and transmission. These 5 priorities share the immune responses of infection with $L$. donovani as an important final common pathway, for which innovative new genomic and non-genomic tools in various disciplines have become available that provide new insights in clinical management and in control. From this, further precision may be defined for groups (e.g. children, women, pregnancy, HIV-VL co-infection), and eventually targeted to the individual level.

Keywords: precision medicine and public heath, visceral leishmaniasis, diagnosis, treatment, PKDL, asymptomatic infection, transmission and infection, immune responses

\section{INTRODUCTION}

Visceral leishmaniasis (VL or kala-azar) is caused by protozoal Leishmania (L.) donovani or L. infantum parasites that are transmitted by sand flies of the Phlebotomus spp. (Old World) or Lutzomiya spp. (New World). There may be anthroponotic and/or zoonotic transmission. The burden of VL is principally in 6 countries (India, Bangladesh, Sudan, South Sudan, Ethiopia, Brazil) (Alvar et al., 2012). While VL is a parasitic disease, virtually all features in clinical presentation, pathophysiology, diagnosis, treatment, outcome as well as epidemiology (herd immunity, family clustering) are determined by the host immune response, that, among other factors, is dependent on the genetics of the host, and in response to the challenge by the causative Leishmania parasite and relevant the sand fly vector (Figure 1). 


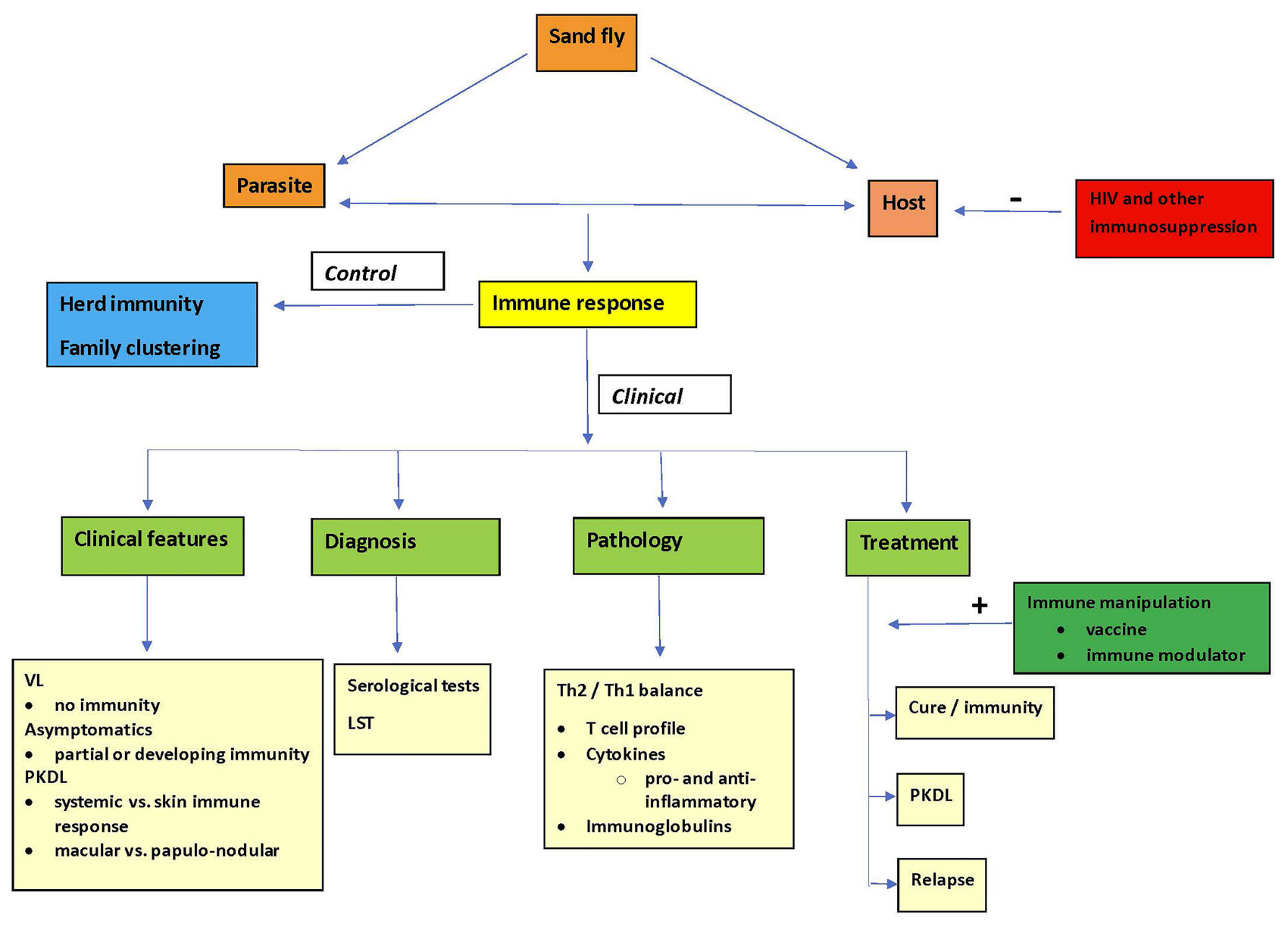

FIGURE 1 | The pivotal role of the immune response in control and clinical aspects of visceral leishmaniasis.

Post-kala-azar dermal leishmaniasis (PKDL) is a skin condition characterized by macules, papules, nodules or polymorphic lesions that develop after successful treatment for VL around persisting parasites in the skin, while systemically parasites may no longer be demonstrable in an otherwise healthy individual. It is an intermediate disease state that precedes complete cure from VL. The clinical features are determined by the immune responses. PKDL is thought to play an important role in transmission and is largely restricted to VL caused by L. donovani (Zijlstra et al., 2003).

In control efforts, there are important regional differences: with the spectacular reduction of VL cases in the Indian Subcontinent (ISC) due to the successful Kala-azar Elimination Program (KAEP), Africa now has equal numbers of VL cases (Rijal et al., 2019; World Health Organization, 2020). In neighboring Ethiopia HIV VL co-infection is common that may contribute considerably to transmission (Diro et al., 2014). It is likely that the approach to control in each endemic area is guided by regional specific factors; there is no 'one size, fits all' in diagnostics, therapeutics or other determinants of control. There are serious gaps in understanding transmission dynamics leading to lack of precision.

\subsection{Precision Medicine and Precision Public Health}

While personalized medicine seeks to utilize treatments and preventive strategies tailored to the individual on the basis of genetic traits only, precision medicine was defined later as the use of diagnostic methods and treatment targeted to the needs of the individual patient(s) on the basis of genetic, lifestyle and environmental determinants.

Human genetics is increasingly contributing to existing research tools, at the level of population, at the level of individual and even to the level of a single-cell e.g. a lymphocyte in relation to a wide array of its immunological properties. This is due the emergence of recombinant DNA and molecular genetic tools including next-generation sequencing of whole genomes and exomes (Neu et al., 2017).

Precision public health (or precision global health) refers to the population perspective and includes applying methods and technologies to measure disease, pathogens, behaviors and susceptibility, as well as developing policies and targeted implementation programs (Singh et al., 2020). Basically, it has been defined as 'providing the right 
intervention to the right population at the right time' (Khoury et al., 2016).

In this paper we introduce the concepts of precision medicine and global health to define new insights in VL clinical management and more efficiency in control efforts (Figure 2).

\section{METHODS}

In absence of publications on precision medicine and global health in VL, relevant literature (general or on VL) was searched for following keywords: genetics, genomics, epigenetics, transcriptomics, nanotechnology, proteomics, metagenomics, pharmaco-genomics, pharmaco-economics, precision medicine and precision global health. The results were first analyzed in factors relating to the host, the parasite and the vector in VL. From this, current and new insights are discussed in the context of existing pitfalls in each of the five main areas relevant for VL clinical management and control: diagnosis, treatment, asymptomatic infection, PKDL and transmission, by discussing new tools (genomic or non-genomic) and re-assessing existing tools. Lastly, a synthesis was attempted to define priorities in precision medicine and global health in VL at the regional level, and where appropriate, at the group or individual level, and suggest the way forward (Flahault et al., 2017; Flahault and Salathe, 2017).

\section{RESULTS AND DISCUSSION}

\subsection{Factors Relating to the Host, Parasite, and Vector}

\subsubsection{The Host}

Genetic studies in VL have indicated that the polymorphic HLADR-DQ region within the major histocompatibility complex of immune related genes is the single major determinant of VL. This was found in a study that assessed three independent cohorts in L. donovani in India and L. infantum/chagasi in Brazil; a similar genome-wide significance was not demonstrated in similar studies in cutaneous leishmaniasis (CL) (Fakiola et al., 2013). In VL, these studies highlighted the importance of antigen presenting cell function and regulation of IFN-y in host-parasite interaction (Fakiola et al., 2013; Blackwell et al., 2020). Interestingly, development of PKDL was linked to the decreased function of the interferon-gamma receptor 1 gene (IFNGR1), that was not found in VL. This was also found in skin biopsies from PKDL patients both from Sudan and India, with uniform low expression of IFN-y and IFNGR1, possibly explaining the persistence of parasites (Ansari et al., 2008; Farouk et al., 2010). Further studies are needed to explain differences within East-Africa and within the ISC, to describe the differences in PKDL rate, clinical presentation and interval between VL and PKDL.

Pharmacogenomics is the study of the response drug therapy in relation to variations of DNA and RNA characteristics;

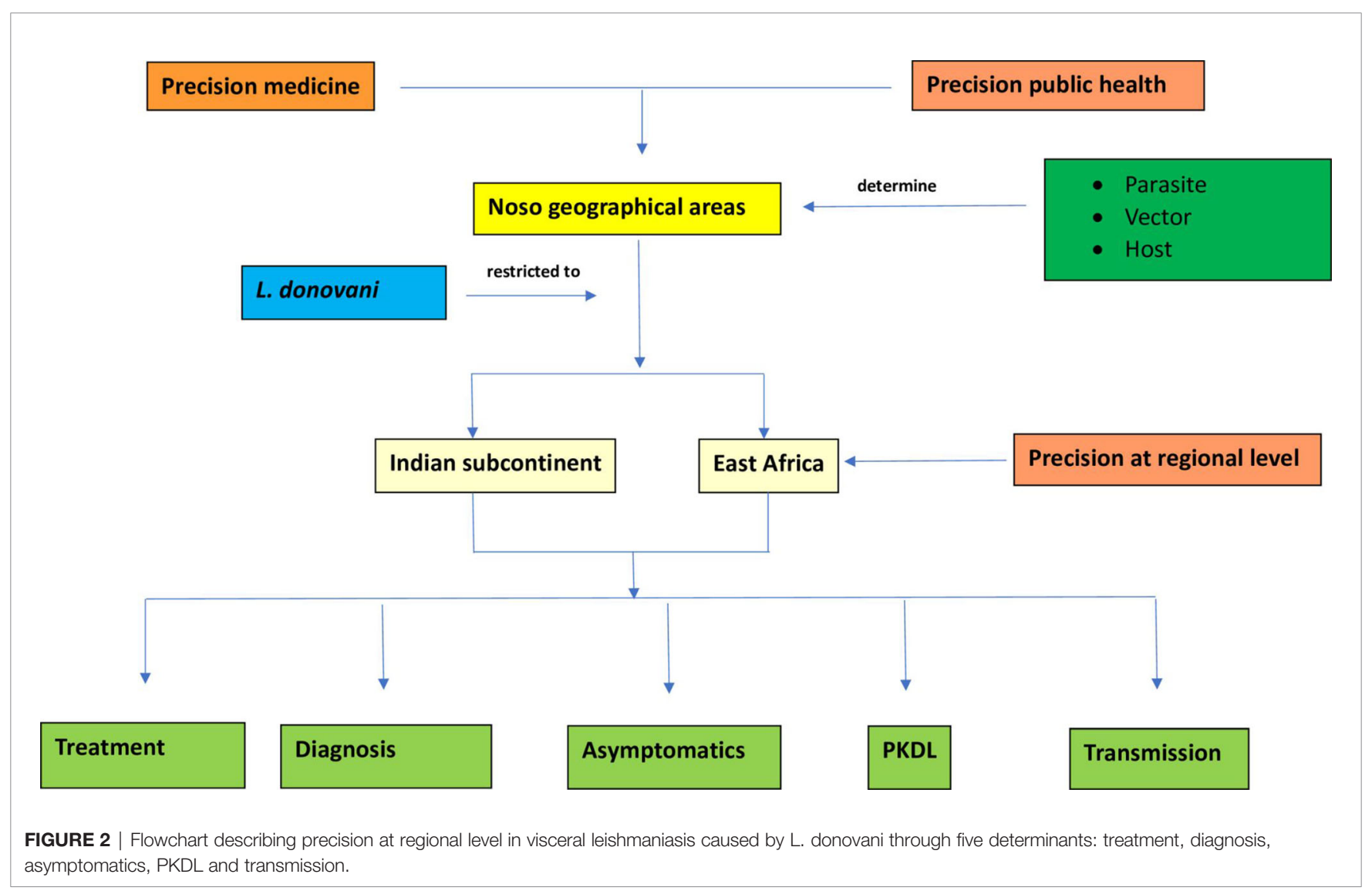


pharmacogenetics studies variations in DNA sequence in relation to drug response (Roden et al., 2019). This may be done by whole genome screening, whole exome screening or custom target sequencing microarrays (Mitropoulos et al., 2015). In Africa, as well as in Asia, genomic analyses have demonstrated that considerable genetic polymorphisms exist in the cytochrome P450 that may translate to differences in drug response (Mitropoulos et al., 2015).

In a more recent meta-analysis on the allele frequencies for 2 common liver enzymes in drug metabolism (CYP2D6 and CYP2C19), a mean risk of an abnormal CYP2D6 or CYP2C10 activity was found to be $36.4 \%$ and $61 \%$ respectively; there was major geographical variation: the range for abnormal CYP2D6 activity varied between 61\% (Algeria) and $2.7 \%$ (Gambia), and for abnormal CYP2C19 between 80\% (India) and 32\% (Mexico) (Koopmans et al., 2021). This is important in the classification of individuals as slow, normal, rapid or ultra-rapid metabolizers. This means that in individuals who are not normal metabolizers, administration of a drug may cause (increased) toxicity or leads to ineffective treatment. For example, codeine is activated by CYP2D6 to its active metabolite morphine; in slow metabolizers this may lead to absence of a drug effect where as in rapid metabolizers it may lead to toxic and dangerous levels of morphine (Roden et al., 2019). Of the currently used anti-leishmania drugs, only pentamidine is metabolized by cytochrome P450 (Kip et al., 2018). Clearly, these concepts are also important for any co-administered drug in VL treatment.

The establishment of a biobank and pharmacogenetics database as for African population facilitates genomic research in diverse populations (Matimba et al., 2008). Fortunately, the cost of sequencing and genotyping is decreasing due to technological advances and national genomics research and policies are within the reach of governments in low-and middle income countries (Tekola-Ayele and Rotimi, 2015). This area of research has been neglected so far, despite inter- and intraregional differences in response to anti-leishmanial treatment.

In addition, more than 300 proteins are involved in ADME (absorption, distribution, metabolism and excretion) of drugs; there is insufficient information on the (variation) of genomic architecture underlying this (Chaudhry et al., 2016). This needs to be further explored in each population affected by leishmaniasis, to predict efficacy and/or toxicity of (new) drugs (Matimba et al., 2008; Baker et al., 2017; Kip et al., 2018). In case of severe toxicity, pharmaco-economics (the economic evaluation of drug administration) may be useful in defining cost-effectiveness of screening prior to administering treatment. For example, testing for glucose-6-phosphate dehydrogenase deficiency (G-6-PD) to avoid drug-induced hemolytic anemia such as caused by primaquine administration in the treatment of P. vivax malaria (Mitropoulos et al., 2015).

\subsubsection{Sex and Gender}

Sex refers to biological differences while gender refers to social differences, relating to specific behavior, activities and roles of men and women in society (Mauvais-Jarvis et al., 2020).
The male to female ratio of VL cases in the Indian subcontinent reported by health facilities (1.40 [95\% 1.37$1.43]$ ) is similar to the risk ratio of incident VL during doorto-door screening (risk ratio 1.27 [95\% CI 1.08-1.51]). Population-based studies showed that males were more likely to be seropositive in the DAT at baseline and more likely to develop VL or remain seropositive. These differences were not found below the age of 15 years (Cloots et al., 2020). Other studies showed that male sex was a risk factor for death or VL relapse, especially after puberty. The role of sex hormones has been established in animal experiments, and hormonal intervention has been suggested. Other factors may be involved such as the causative parasite as male risk is increased in L. donovani infection, but not in L. tropica (Lockard et al., 2019).

The influence of sex hormones on modulation of the immunity is poorly investigated; this may affect differences in outcome between males, and females, as well as non-pregnant and pregnant females. Males are more commonly affected with more serious clinical features, and it thought this is true despite better access to health care and more exposure to sand flies (Snider et al., 2009).

\subsubsection{Co-Infection}

Protective immune responses in leishmaniasis are required for strong and long lasting immunity by persistent parasites through concomitant immunity (Mandell and Beverley, 2017). In patients who are cured, persistent replication takes place, mostly in activated antigen-presenting cells. In L. major infection, these are at the site of infection and draining lymph nodes and stay there for the rest of the host's life (Okwor and Uzonna, 2008). It follows that any interference either by an infective agent or drug treatment may result in reactivation and clinical recurrence.

VL may occur with co-infections of other common (tropical) conditions, of which HIV infection and malaria are most common.

Immunosuppression is a risk factor for VL; this includes HIV infection, the use of immunosuppressant drugs (including steroids), haematological malignancies and patients with transplants (van Griensven et al., 2014). HIV co-infection causes increased susceptibility of infection, more severe disease, and diagnostic and therapeutic problems. This was also described for PKDL (El Hassan et al., 2013; Zijlstra, 2014). In IV drug addicts, a second mode of transmission cycle may exist between humans through exchange of contaminated needles. Conversely, Leishmania infection influences the clinical course of HIV infection (van Griensven et al., 2014). Tuberculosis may occur in VL-HIV co-infection influencing outcome (van Griensven et al., 2018). PKDL and VL with concomitant skin lesions are common and more severe in HIV co-infection (Zijlstra, 2014). Similarly, malnutrition may aggravate (HIVrelated) immunosuppression, but its role is unclear as weight loss as a clinical feature may be caused by VL (Akuffo et al., 2018).

A different mechanism was described in the treatment of hepatitis C with Direct Acting Antivirals (DAA) that downregulate interferon and interferon receptors, thus leading to re-activation of previous leishmania infection (Colomba et al., 2019). 
Helminth infection affects up to one-third of the global population and cause chronic infection with downregulation of the pro-inflammatory immune response an enhanced Th2 response and repair mechanisms. Chronic helminth infection suppresses antibacterial, antiviral and antiprotozoal immunity, leading to increased susceptibility and attenuated immunopathology (McSorley and Maizels, 2012). This may be modulated by the excretory and secretory products (the secretome) (Ditgen et al., 2014). The effect on susceptibility and severity of a concomitant disease may be aggravated by helminth-induced anemia and malnutrition (Ditgen et al., 2014). Currently, there is no evidence that helminth infection predisposes to more severe disease (in VL) or treatment failure of stibogluconate (in CL) (Tajebe et al., 2017; Martinez et al., 2020). The role of helminth infection as a trigger to develop PKDL has not been studied. Gut bacteria were also shown to be possibly implicated as VL patients showed dysbiosis of gut flora compared to healthy controls (Blackwell et al., 2020).

\subsubsection{Unexplored Co-Infection}

The role of co-infection remains largely unexplored; measles may be taken as an example that continues to be a world-wide problem despite the availability of a vaccine. While natural infection induces a strong cellular and humoral immune response, after cure a transient immune suppression with increased morbidity and mortality may occur (Griffin, 2010).

Recently, it was shown that measles virus infection has an impact on circulating lymphocyte subsets, with memory $\mathrm{T}$ and $\mathrm{B}$ cells being most severely affected, causing loss of immunological memory and renewed to susceptibility to pathogens (Mina et al., 2019). Childhood vaccinations such as for measles are associated with reduced risk of measles (Lima et al., 2018). It has been suggested that measles could lead to breakdown of immunity; in a case report recurrence of VL after PKDL occurred after measles infection. Similar co-infections that may reduce immunity are malaria, tuberculosis and Epstein-Barr virus infection (Louzir et al., 1993; Nandy et al., 1998). There are no formal studies that examine the effect of measles or other common viral infections such as hepatitis B and hepatitis C on (recurrence of) VL or the occurrence of PKDL after seemingly successful treatment of VL. Concomitant malaria in VL was found to be associated with a more severe clinical picture of VL, including severe anemia, and increased mortality in Sudan (van den Bogaart et al., 2013).

\subsubsection{Genetic Determinants of Response to Infection}

Transcriptomics describes the immunological response to infection by assessing the gene expression ('signature') in the interaction between host and pathogen. Using whole blood transcriptional profiling, the gene expression profile of active VL was demonstrated to be different compared with healthy controls, but this was not found in asymptomatic infection and healthy controls. In addition, support was found for more effective cure at 30 days post treatment for single dose AmBisome vs. multi-dose conventional amphotericin B for 30 days (Fakiola et al., 2019). In a recent study, an analysis of the whole blood transcriptome in HIV-VL co-infected patients identified 4 genes that accurately determined the treatment outcome (Adriaensen et al., 2020). This is a promising tool that needs further exploration, particularly to the correlation with actual immunity and immune parameters thereof in various clinical settings and endemic regions.

\subsubsection{The Parasite}

Identification of the causative parasite in each clinical and epidemiological setting is crucial. Issues in taxonomy and species typing have been thoroughly reviewed (Van der Auwera and Dujardin, 2015).

While L. donovani causes VL both in Asia and Africa, molecular analysis of the parasite shows that there are three clusters: one East African cluster (Sudan, Ethiopia), a second with mixed Indian and Kenyan isolates and a third with L. infantum (Kuhls et al., 2007; Lukes et al., 2007; Jaber et al., 2018). It is not clear to what extent these differences explain different PKDL rates, performance of serological (rapid) diagnostic tests or response to treatment, and these should be understood in relation to the host immune response.

Molecular typing of the parasite has provided insight in the differences in epidemiological, clinical features within east Africa where L. donovani is the common causative agent in the whole region. In Sudan/North Ethiopia (NE group), the sand fly is $P$. orientalis, the $\mathrm{PKDL}$ rate is high and the response to paromomycin $<67 \%$, while in contrast, in South Ethiopia (SE group), the sand fly is P. martini and the PKDL rate is low, with better cure rates for paromomycin (>87\%) (Hailu et al., 2010; Zackay et al., 2018). Whole genome analysis has demonstrated a strong correlation between one single nucleotide polymorphisms (SNP) in L. donovani and NE and SE groups, with genes involved in parasite viability and resistance to drugs (Zackay et al., 2018).

The methodology used is important; while southern blotting using a DNA probe to the L. donovani 28s rRNA gene did not show difference between NE and SE, amplification fragment length polymorphisms (AFLP) grouped the strains according to geographical origin with further subdivision in subpopulations by zymodeme, geography and year of isolation, but not by clinical features. Interestingly, skin isolates had fewer polymorphic AFLP fragments than VL strains, but strains of most VL and PKDL patients grouped together, suggesting that unidentified genetic factors may underlie development of PKDL (Jaber et al., 2018). Another study found polymorphism in the HASPB repeat region (also known as k26) of east African $L$. donovani strains (north Ethiopia, Sudan and Kenya) which could influence the performance of a diagnostic test or a vaccine based on k26 (Zackay et al., 2013).

In Brazil, natural resistance of $L$. infantum to miltefosine was demonstrated in VL patients who failed to respond to treatment and was linked to a Miltefosine Sensitivity Locus (MSL) by the use of Genome-Wise Association Study analysis of SNPs, gene and chromosome copy number variations (Carnielli et al., 2018; Carnielli et al., 2019).

In the ISC, L. donovani causes VL while in Sri Lanka, it also causes CL. A recent study suggested that SNPs and variable gene expression causes differences in tropism (Samarasinghe et al., 2018). 
Other genomic studies have demonstrated that $L$. donovani strains may form a genetically distinct population that are the basis of resistance to stibogluconate, rather than SNPs (Samarasinghe et al., 2018).

Also, in the ISC, there is no convincing evidence that strains causing VL and PKDL differ, but there are few data on paired strains and most studies are cross sectional. In addition, only minor differences in strains from VL and PKDL have been demonstrated so far, except for drug sensitivity (Dey and Singh, 2007; Subba Raju et al., 2008; Mishra et al., 2013). However, some proteins are upregulated such as Gp63 and PSA, that may alter the parasite and promote accumulation in the skin (Salotra et al., 2006). Parasite strains from Sudan examined by PCR single strand conformation polymorphism did not show correlation with clinical manifestations (Bermudi et al., 2020). VL and PKDL strains clustered in the same branches of the tree examined by multi-locus microsatellite typing (MLMT) or multi-locus sequence typing (MLST) (Alkhaldy et al., 2020; Bolia et al., 2020).

\subsubsection{Co-Infection With Endosymbionts}

Leptomonas seymouri is an opportunistic parasite that is assumed non-pathogenic to humans but may be an opportunistic pathogen in HIV infection. It has also been demonstrated in VL and PKDL patients and HIV infected patients with diffuse cutaneous leishmaniasis (DCL) (Dedet et al., 1995; Ghosh et al., 2012). Co-infection was demonstrated in $13.8 \%$ of patients with VL/PKDL caused by L. donovani in India. The implications hereof are not clear, including a role in antimony unresponsiveness to stibogluconate (Ghosh et al., 2012). Recently, it was also demonstrated in cutaneous leishmaniasis caused by L. donovani in India, suggesting a role in pathogenesis as L. donovani generally causes visceral disease. It has also been demonstrated in sand flies. There is similarity in the genome of leptomonas and Leishmania. The biology and role of leptomonas as a co-infecting parasite or a hybrid of Leishmania needs further investigation (Thakur et al., 2020).

Species of Leishmania may show the presence of Leishmania RNA virus (LRV), member of the Totiviridae, that has been associated with treatment failure and relapse in CL caused by L. braziliensis and L. guyanensis infection (Adaui et al., 2016; Macedo et al., 2016). Its role remains controversial; a recent study in CL caused by L. guyanensis did not show a relationship between treatment failure to pentamidine treatment or the presence or genotype of LRV (Ginouves et al., 2021). In another study, it has been associated with development of Diffuse Cutaneous Leishmaniasis (DCL) (Rossi and Fasel, 2018). LRV viral DNA interacts with the Toll-like receptor 3 (TLR3) on the macrophage and triggers the production of proinflammatory cytokines including interferon- $\beta$ and thus confers a selective advantage to leishmania, include its retention and capacity to metastasize (Grybchuk et al., 2020). Its action is through production of type I interferons suggesting that other viruses may have a similar effect (Rossi et al., 2017). A first nonLRV RNA Leishmania-infecting leishbunyavirus has been described (Grybchuk et al., 2020).

\subsubsection{The Sand Fly Vector and Transmission}

Major progress has been made to describe the development of the leishmania parasite in the sand fly and factors determining transmission during a sand fly bite. Genetic exchange between Leishmania parasites occurs within the sand fly contributing to phenotypic diversity that influences resistance, virulence and tropism (Akopyants et al., 2009). In addition to leishmania parasites, sand flies may be infected and transmit viruses such as phleboviruses. Preliminary reports suggest that infection by Leishmania and (phlebo) viruses is not compatible in the sand fly midgut and may affect leishmanial development; this could be further studied in the context of mode of Leishmania control (Telleria et al., 2018). Similarly, Leishmania development in the sand fly midgut may be inhibited by other microbiota such as bacteria and fungi that are co-egested with Leishmania during the blood meal and offer possibilities for paratransgenic or biological control as a prophylactic or therapeutic option (Campolina et al., 2020; Karmakar et al., 2021). In the mouse model these microorganisms may facilitate the establishment of Leishmania infection by activating the neutrophil inflammasome with production of IL-1b (Dey et al., 2018). Sand fly saliva is equally co-egested with the Leishmania parasites during a bloodmeal and has anti-hemostatic, anti-inflammatory, and immunomodulatory properties. In the naive host sand fly saliva promotes Leishmania infection with larger numbers of parasites and larger lesions. Repeated exposures result in protection as the host is immunized by salivary proteins. This potential is supported as bites from non-infected sand flies outnumber those from infected sand flies (Karmakar et al., 2021). These antigens may be exploited in vaccine development, whereas anti-saliva antibodies may be used in epidemiological studies (Lestinova et al., 2017). The role of saliva from vectors other than sand flies merits further study (Karmakar et al., 2021).

\subsection{Impact of New Genomic and Non- Genomic Tools on Insights to Precision and Identification of Priorities}

\subsubsection{Diagnosis}

Current pitfalls in diagnosis include the use of highly sensitive diagnosis by molecular tools for demonstration and typing of the parasite in various tissues including peripheral blood, replacing classical microscopy and culture. Second, serological tests have poor specificity as antibodies may persist from previous leishmanial infection. Third, molecular parasitological methods may not be as useful as test of cure (biomarker) as immunological parameters that reflect the developing immune response rather than disappearance of the parasite (Salotra et al., 2001; Antinori et al., 2007; Boelaert et al., 2014; Sundar and Singh, 2018; Zijlstra, 2019).

\subsubsection{Demonstration of Parasites}

Demonstration of parasites by microscopy is still considered the gold standard in diagnosis of VL and PKDL in many endemic areas, and the preferred method in patient care, particularly in Africa, or in research projects. Microscopy requires training and 
a working microscope; the parasite load may be quantified, e.g. in a spleen aspirate (Sokal, 1975). All patients are treated with the same regimens independent of the parasite load; precision to the individual level has not been studied. Digital imaging and reading of slides is under development and allows reading with adjustment of focus and magnification, and may be used for quality assurance. In malaria, for example, a timed tally counter for reading thick blood films, was found to provide richer, more accurate data, and less time consuming (Nuel and Garcia, 2021). So far, no studies have been published in leishmaniasis.

Antigen tests differ in sensitivity and specificity with lowest and highest sensitivities are reported from Nepal/Tunisia and Europe/Middle East respectively $(\mathrm{p}<0.05)$, and the lowest and highest rates of specificity were reported from Sudan and America/Middle East, respectively (Fararouei et al., 2018).

Microscopy and culture are gradually superseded by molecular tools, such as PCR; a pan Leishmania PCR may be done or a species-specific PCR particularly if a certain subspecies is suspected. Subgroups may be identified by various molecular methods (Van der Auwera and Dujardin, 2015). PCR is usually done on aspirates of lymph node, bone marrow or spleen aspirate, with increasing sensitivity reaching $95-97 \%$ in a spleen aspirate (Zijlstra and el-Hassan, 2001). In HIV-VL infected patients, PCR in blood may be used (Antinori et al., 2007). Antigen tests should also be carefully evaluated against the superior sensitivity of PCR based diagnosis and test of cure.

Challenges include the use of PCR in the field; using modifications to classical PCR such as LAMP and recombinase PCR can be done in the suit-case field laboratory (Verma et al., 2013; Mondal et al., 2016). For differential diagnosis, a multiplex PCR may be developed; this would be a simultaneous test for malaria, schistosomiasis, or brucellosis, or co-infection with HIV according to the local epidemiology; this requires regional precision.

Parasite detection by PCR as a test of cure indicates disappearance of the parasite but not necessarily a protective immune response. qPCR offers quantification of the parasite load and may be useful as a biomarker (Wu et al., 2020). Recently, qPCR in blood of non-HIV infected VL patients 2 months after start of treatment was shown to correlate well with tissue parasite load and predict treatment response (relapse) in East Africa (Verrest et al., 2021).

\subsubsection{Antibody Tests}

In VL, a strong humoral response exists as evidenced by polyclonal gamma-globulinaemia; this consists mainly of IgG antileishmanial antibodies that may be used in serodiagnosis, reflecting largely the Th2 immunological response.

Multiple serological antibody tests have been designed and studied in the past decades; these mainly include the direct agglutination test (DAT), rK39 ELISA and IFAT. The sensitivity is satisfactory, but a major problem exists with specificity. While this may be influenced by cross-reaction with other antigens, in most cases antibodies that are detected may result from previous exposure to Leishmania (clinical or subclinical) as these antibodies may persist for many years (Khalil et al., 2013). Often sensitivity and specificity are determined by testing stored sera, using healthy endemic controls or sea from patients with other diseases such as malaria. It is however essential to evaluate a diagnostic test in a prospective study, with proper follow-up of those tested positive without clinical features, to establish the outcome and the measure positive and negative predictive value (PPV and NPV, respectively). It should be noted that the PPV may vary according to prevalence of the disease and perform better when transmission is high, such as during an outbreak.

The rK39 rapid diagnostic tests (RDT) is the only RDT that has been successfully developed in VL; it was found $90 \%$ sensitive and $100 \%$ specific in India, during a time of high prevalence of $\mathrm{VL}$, and the test is used in the KAEP in the ISC (Kumar et al., 2001). However, false positive results may occur in malaria, tuberculosis, liver cirrhosis and chronic myeloid leukaemia (Topno et al., 2020). In a Cochrane review, the rK39 immunochromatographic test (RDT) showed overall sensitivity in the ISC was $91.9 \%$ (95\% CI 84.8-96.5) vs $85.3 \%$ (95\% CI 90.099.5\%) in East Africa. In East Africa, the sensitivity was lower possibly because of lower antibody production below the cut-off point for detection that may be a function of parasite virulence (Zijlstra et al., 1998; Boelaert et al., 2014). This issue shows the precision needed at regional level. All other tests lack accuracy, validation, or both (Boelaert et al., 2014). A re-evaluation of the performance of the rK39 RDT (positive predictive value) in the KAEP may be useful, as the prevalence of VL has dropped dramatically. (www.who.int/en/newsroom/factsheets/detail/ leishmaniasis). Other antibody tests are based on rK9 and rK26 antigens; these were shown to have lower sensitivity than rK39 in India (Mohapatra et al., 2010). Studies in other regions are awaited.

Targeting multiple antigens may be useful to increase test performance. Recently, a monoclonal antibody-based multiplex capture ELISA with five different monoclonals to leishmania proteins was found to have improved sensitivity of $\geq 93 \%$ and $100 \%$ specificity in urine samples from Brazil and Kenya (Abeijon et al., 2020).

\subsubsection{Immunological Tests}

The cellular immune responses in VL and PKDL have been recently reviewed (Zijlstra, 2016). For convenience, the immune response is described in terms of a Th1 and Th2 response; while in the animal model this dichotomy is genetically determined, this has not been demonstrated in humans where markers of both Th1 and Th2 response co-exist in VL and vary during treatment or in the natural history (Zijlstra, 2016).

In VL, the Th2 response is characterized by the absence of a cellular immune response against Leishmania parasites: peripheral blood mononuclear cells (PBMC) do not proliferate in response to stimulation with leishmanial parasites; in vivo the leishmanin skin test (LST) is typically negative. While there is a predominant role for anti-inflammatory cytokines (Th2 response) such as IL-10 and TGF- $B$, also high levels of interferon- $\gamma$ (INF- $\gamma$ ) can be demonstrated that is typically associated with healing (Th1 response) suggesting that there is no Th1 defect in active VL. This shows the complexity of the immune response that cannot be captured in a Th1 and Th2 dichotomy alone (Faleiro et al., 2014). Various other mediators 
need to be described according to disease stage, severity and outcome (Brodskyn and Kamhawi, 2018).

Analysis of cytokines responses requires an advanced laboratory. Recently, a novel point-of-care device was presented that directly monitored a panel of five cytokine markers that may reflect the host's immune response to sepsis. It used a sensor disposable cartridge attached to an electrochemical reader. This could have great potential in VL and PKDL as a marker of infection and as a biomarker to monitor the response to treatment (Tanak et al., 2021).

Cytokine panels may identify crucial levels or a ratio of key anti-inflammatory and pro-inflammatory cytokines that correlate with diagnosis, improvement during treatment, cure in VL as well as prediction of PKDL. Such panels have the optimal potential to also serve as a biomarker; it should be tailored to precision at the regional, or intra-regional level.

New approaches that measure genetic determinants of infection such as a gene signature in a transcriptomics approach are promising (Fakiola et al., 2019; Adriaensen et al., 2020).

The Leishmanin skin test (LST) (commonly called the Montenegro test in Latin America) measures type IV cellmediated immunity. Intracutaneous injection of killed leishmania parasites induces induration that is measured after 48-72 hours (Sokal, 1975). Normally, the LST is negative in VL, may be positive or negative in PKDL and becomes positive after cure (Zijlstra and el-Hassan, 1993). There is no consensus about a cut-off size of induration for a positive test; while classically 5 $\mathrm{mm}$ is used, others consider any induration a positive result.

The LST is easy to use and may obviate the need for testing immune responses in the peripheral blood. It identifies susceptible individuals as these are LST negative; a positive LST indicates previous exposure (clinical or subclinical) and is generally considered a parameter of a Th1 response and an indication of immunity (Gramiccia et al., 1990; Zijlstra and elHassan, 1993; Schaefer et al., 1994). It is however not clear if a positive LST result indicates life-long protection and whether repeated exposure is required. In immunosuppression a positive test may become negative which correlates with increased chance of re-infection or recurrence of a previous infection.

The LST is a powerful epidemiological tool. Its use may be demonstrated in control programs such as the KAEP in the ISC to monitor herd immunity once transmission is under control; similarly a study in Sudan demonstrated that a positive LST was associated with protection for VL (Zijlstra and el-Hassan, 2001). Alternatively, it is useful to screen for non-immune individuals in a prophylactic vaccine trial (Khalil et al., 2000; Regional Strategic Framework for Elimination of VL From SEA Region (2005-2015)). In other epidemiological cross sectional studies, LST positivity rates were not consistent and it is not certain if this is due to lack of boosting by repeated exposure to sand fly bites or to the poor performance of the LST antigen used (Bern et al., 2006).

Currently there is no LST antigen available because of issues, among other, in Good Manufacturing Practice (GMP). L. major and L. infantum antigen have been used most and were satisfactory in detecting previous L. donovani infection (Zijlstra and el-Hassan, 1993). L. amazonensis antigen has also been used with better sensitivity than L. infantum (Bern et al., 2006). The LST, provided it is manufactured by GMP, may still be a useful and powerful epidemiological tool in control programs, to assess immunity in the individual and in the population; it will need to be assessed for accuracy in different populations. There is an urgent need for a new LST manufactured according to GMP, with L. donovani antigen; research efforts are on-going. (https:// www.ghitfund.org/investment/portfoliodetail/detail/159)

Metagenomic next-generation sequencing (mNGS) may offer opportunities for screening for unexpected co-infection. It complements other (routinely used) molecular based diagnostics as it aims to identify any nucleic acid in the samples in an unbiased way and thus may identify organisms that not suspected and otherwise cannot be cultured. It also provides information on subspecies and antimicrobial susceptibility. Disadvantages include contamination and lower sensitivity for organisms with small genomes or present in low quantities (Ko et al., 2019; Li et al., 2021). While this technology has the advantage of diagnosing coinfections, the significance of finding a second microbe should be carefully assessed as to its clinical relevance. It has been used in leishmaniasis on e.g. bone marrow aspirate and trephine for identification and speciation of Leishmania (Williams et al., 2020). This option may be further explored in the differential diagnosis of infectious diseases, using more accessible samples such as blood in VL or a skin slit smear in PKDL. New portable and affordable devices may be introduced in resource-limited settings (Lu et al., 2016). Other applications include a metataxonomic analysis of gut microflora using 18S rRNA gene sequencing that showed dysbiosis of bacterial diversity in the gut of VL patients compared with healthy controls; this could be explored further (Blackwell et al., 2020). Metagenomic studies may be used to address unsolved issues in co-infection and the effect thereof in immune responses, including the late occurrence of PKDL in the ISC.

Nanotechnology (nanodiagnostics) is based on biomedical detection systems that do not require a read-out tool such as a gel, PCR or culture; they are rapid and seem cost-effective for use in the field. In VL, the potential for use in an elimination program was suggested because of superior sensitivity for early detection and simultaneous detection of drug resistance (Singh et al., 2019; Gedda et al., 2021). Theragnostics combines diagnostics and therapeutics using nanotechnology and holds promise for accurate monitoring of infection in the field. Rather than measuring gene polymorphism as in pharmacogenomics, it is more flexible and may use combined information from genomics, proteomics and metabolomics (Ozdemir et al., 2006).

\subsubsection{Treatment}

\subsubsection{Treatment of $V L$}

Developments in drug treatment in the past decades have focused on drug resistance (sodium stibogluconate, SSG), the first oral drug (miltefosine), combination therapy and targeted drug delivery (AmBisome). All are primarily aimed on elimination or reduction of parasites leading to cure and 
preventing relapse. Pitfalls in treatment of VL were summarized in a recent review (Alves et al., 2018). These include regional and intra-regional differences in cure rates of anti-leishmania drugs, resistance, the need for combination treatment, risk of PKDL and adequate dosing guided by pharmacokinetic studies.

There are important regional differences. In some these differences seem related to the drug used and host; AmBisome and miltefosine have lower cure rates in Africa compared with the ISC (Musa et al., 2010; Wasunna et al., 2016). This has led to the adoption of short course SSG + paromomycin combination therapy for 17 days as the standard treatment; this combination was found to be non-inferior was demonstrated to 30 days of SSG, thus decreasing SSG related toxicity (Musa et al., 2012). In the KAEP in the ISC single dose AmBisome is one of the pillars to success (WHO, 2011; Musa et al., 2012).; second line treatment may be with AmBisome combined with miltefosine and paromomycin, respectively (Sundar et al., 2011). While SSG is no longer used in the ISC because of resistance, this is not reported from East-Africa. The poor performance of miltefosine in VL caused by in Brazil was linked to the parasite (L. infantum) (Carnielli et al., 2018).

Miltefosine was found to fail in the treatment of VL in children as a result of variable drug exposure. In a recent trial on treatment of children with VL, linear dosing was replaced with allometric dosing with less variable exposure and increased efficacy, thus providing precision to group level (Mbui et al., 2019).

The treatment of VL-HIV co-infection was recently studied; a combination of high dose AmBisome (30 mg/kg total dose) and miltefosine $100 \mathrm{mg}$ daily for 30 days was found superior to monotherapy AmBisome (40 mg/kg total dose), showing that precision may be applied to the group level (those who are HIV infected), while confirmation is awaited from other studies in the ICS (Diro et al., 2019).

\subsubsection{Risk of $P K D L$}

Drug treatment of VL is an important factor in the incidence of PKDL. In a follow-up study in Bangladesh of VL patients treated with monotherapy (SSG, miltefosine, paromomycin, single dose AmBisome [SDA], multidose Ambisome), or combination therapy (AmBisome + miltefosine, AmBisome + paromomycin, miltefosine + paromomycin), it appeared that SSG and multidose AmBisome had significant lower PKDL and relapse incidence rates than the other regimens (Mondal et al., 2019). In a similar study from India, SDA had a lower PKDL risk than miltefosine + paromomycin, with higher risk in females and children $<12$ years. Children also had a higher risk of relapse (Goyal et al., 2020).

In Africa, PKDL incidence was found to be high in those treated with poor drug treatment (low dose, incomplete courses, poor quality SSG), and in young children. Treatment with paromomycin lead to higher PKDL rate in those treated with low dose of longer duration (Hailu et al., 2010).

Recently, the clinical pharmacokinetics of systemically administered anti-leishmania drugs was reviewed (Kip et al., 2018). While PK data have been described for pentavalent antimony, miltefosine and paromomycin, there are no data on AmBisome and pentamidine. Only miltefosine has been studied to define target exposure levels to effect. Few data exist on paediatric populations and female patients who are pregnant or breastfeeding. Here, precision to the individual (group) level is needed. In HIV-VL co-infection, drug-drug interactions, renal clearance, and protein-binding should be taken into account; conversely, the PK of antiretroviral drugs has not been evaluated in VL (Kip et al., 2018). The importance of pharmacokinetics was recently demonstrated in HIV-VL co-infected patients where low drug exposure of AmBisome and miltefosine was found, possibly explaining the high relapse rate in this patient group (Kip et al., 2021).

\subsubsection{Vaccination and Immune Manipulation}

Leishmanization is the oldest and best form of immunization so far. Inoculation with live virulent $L$. major parasites induces a lesion that fades after 6 months. It was abandoned since inoculation did not induce a lesion in everyone. As the vaccine contains live parasites, it is not stable and thus not practical.

The first vaccines used autoclaved whole parasite leishmania strains such as L. major or L. mexicana; later specific parasite antigens were used. This was followed by vaccines that used antigen delivery through a viral vector, or that used modification or deletion of genes. Various adjuvants and immune-enhancers have been used, including bacille Calmette-Guerin vaccine (BCG) (Zijlstra et al., 2020).

In the only prophylactic trial in VL, using an autoclaved L. major vaccine, no adequate protection for VL was found in Sudan (Khalil et al., 2000). The same vaccine was found to be efficacious in immuno-chemotherapy in patients with chronic PKDL, resulting in LST conversion and increased IFN- $\gamma / \mathrm{IL}-10$ production (Musa et al., 2008).

In addition, research is on-going on an immunomodulator (CpG oligodeoxynucleotide) that stimulates plasmacytoid dendritic cells; it induces maturation of monocytes into mature dendritic cells and is a strong Th1 adjuvant. It is under development by DND $i$ in collaboration with the US Food and Drug Administration. (www.dndi.org).

\subsubsection{Way Forward}

The outcome of VL is determined by the balance between antigen-specific anti-inflammatory cytokine and proinflammatory responses that are determined by host genetic factors (Blackwell et al., 2020). Differences in drug responses should be understood in each noso-geographical region with regard to cure, relapse and $\mathrm{PKDL}$ rates after each $\mathrm{VL}$ drug regimen, in relation to population, parasite and sand fly characteristics. In this respect, it should be noted that not all these issues have been rigorously studied with comparable methodology in all regions. In each of these different outcomes, genetics and immunological factors play a role. In addition, as VL patients are immunocompromised, secondary infections and malnutrition may influence outcome.

Developing new drugs requires a lengthy and costly investment with an uncertain outcome. New candidate molecules are often being explored based on the killing potential of leishmanial parasites in vitro, while the immune response induced by drug treatment is the most important 
indicator for treatment success or failure. This is often not addressed or appreciated until well into the clinical stages of development, in phase II and III studies.

In addition, each drug may have intrinsic immune effects that have not been sufficiently described (Ansari et al., 2008; Mukhopadhyay et al., 2012; Ghosh et al., 2013). This was recently reported in detail for miltefosine that induces enhanced Th1 cytokine responses (Palic et al., 2019). This shows that drug development should be driven by outcome (immunity) rather than parasite killing ability alone. Sterile immunity does not exist and parasites persist under the control of the immune response. Parasite eradication may be detrimental to the host's immunity and thus to herd immunity (Okwor and Uzonna, 2008; Mandell and Beverley, 2017).

AmBisome is the prototype drug for VL targeted drug delivery as it is phagocytosed by (infected) macrophages and accumulates in liver and spleen, with reduced renal and infusionrelated toxicity compared to amphotericin de-oxylate. Nanotechnology (nanoparticle-based drug delivery) holds promise to further refine targeted drug delivery with the potential to decrease drug regimens, drug resistance, drug toxicity, and cost. Further research is on-going which should address nanotoxicity, biodistribution and post exposure biopersistence, as well as theragnostics (Singh et al., 2019).

Randomized Clinical Trials (RCTs) provide precision but are precise for a subset of the population as even with randomization, participation is not uniform and do not take into account other health determinants such as social and demographic factors; these include women (pregnant or in child-bearing age) and children. It follows that all RCTs should be performed in each endemic region in all patient groups affected to address these factors. For this purpose, these should include a diagnostic study to detect co-infections, a study of biomarkers, a PK/PD assessment, pharmacogenomics, and analysis and genotyping of the circulating parasites. A study design in which patients are stratified according to parasite load and outcome may provide precision of treatment to the individual level. The recent study on allometric dosing of miltefosine in children emphasizes the precision needed in patient groups that are not primarily included in RCTs, such as children; the same would apply to (pregnant) women (Mbui et al., 2019).

A number of checkpoint molecules have been identified that provide opportunity for targeted intervention; these are present on CD4+ cells in VL patients, including CTLA-4 (CD152) and $\mathrm{PD}-1$, which negatively regulate T-cells. CTLA-4 binds to costimulatory ligands B7-1 and B7-2; activation of CTLA-4 leads to increased levels of TGF- 3 . PD-1 binds to PD-1 ligand 1 and blockade of either promotes parasite clearance and increased pro-inflammatory cytokine production in experimental VL (Kumar et al., 2017).

Population pharmacokinetic modelling offers insight in drugs with large variability in exposure and the use of allometric dosing; it has the advantage that sparse and heterogenic sampling may be used and this would be suitable for research in remote settings. For this, blood samples collected as dried blood spots are attractive. Special areas of interest are intracellular drug levels and drug levels in the skin vs. systemic levels (Kip et al., 2018).

Investment in drug development needs to be balanced, for example, against investing in vaccine development. Vaccination provides better precision than drug treatment as it aims directly at inducing a protective immune response. Pharmaco-economic assessment may indicate that investing in vaccine development may ultimately be more efficient and more cost-effective in control programs.

\subsubsection{PKDL}

Current issues in PKDL include the inter- and intra-regional differences in pathophysiology, management and prevention (Zijlstra et al., 2003; Zijlstra, 2016; Moulik et al., 2018; Zijlstra, 2019).

\subsubsection{Pathophysiology}

In Sudan, the natural history of VL and PKDL shows that $85 \%$ of PKDL cases self-cure, while $15 \%$ have persistent PKDL and require treatment. An inverted relationship exists between positive serology (DAT titer) and the LST positivity. Those who showed self-cure were more likely to have a positive LST and were less likely to have a positive DAT result. The opposite was found for those who developed persistent PKDL (Musa et al., 2002). Treatment of PKDL in Africa has been poorly studied; regimens include SSG + PM for 17 days or AmBisome for 10 days (Musa et al., 2005; Younis et al., 2016). Other than in Sudan, where PKDL occurs in the context of the developing immune response after seemingly effective treatment of VL, in the ISC the role of an intercurrent infection should be explored; this could be targeted to suspected microbes such as measles or malaria; alternatively, a metagenomic approach may help to identify thus far undetected concomitant microbes.

PKDL patients are different from VL patients as PKDL occurs at a younger age and PKDL is limited to the skin without systemic disease; the patients are on average healthy, not malnourished, and normally do not have anaemia or low albumin levels (Zijlstra et al., 2003). Also here the developing immune response determines the clinical differences and precision in treatment is needed within the spectrum of L. donovani infection based on PK/PD studies. Paired VL and PKDL strains from the same patients and strains from $\mathrm{VL}$ patients who do not develop PKDL should be analyzed to explore if genetic factors in parasites underlie development of PKDL.

\subsubsection{Management}

Diagnosis of PKDL requires parasitological confirmation as the differential diagnosis may be difficult and in the ISC all patients are treated with miltefosine for 3 months. (q)PCR on a slit smear is more sensitive than microscopy (Verma et al., 2013). Recently, a microbiopsy device was found to accurately quantify the parasite load in the skin in PKDL (Cloots et al., 2021). The extent of the rash and at diagnosis and during treatment may be described clinically by grading, using a maniken, clinical photography or a combination of a clinical and parasitological 
score. There are no reliable parasitological, laboratory or immune biomarkers (Zijlstra, 2019). Molecular diagnosis may be done in the field using the mobile suitcase laboratory (Mondal et al., 2016). Novel 3-dimensional optical scanning is a novel tool to measure PKDL lesions with great accuracy with regard to diameter, surface and volume as was demonstrated from Sudan. It is suitable for use under field conditions (Zijlstra et al., 2020).

Diagnosis at field level should be further developed with regard to include the use of multiplex PCR panels in differential diagnosis to detect other infectious causes of a similar skin rash, such as leprosy and fungal infections (e.g. tinea). The use of the field laboratory may be expanded. The use of tele-medicine, artificial intelligence and diagnostic algorithms may be explored further to include all infectious and noninfectious differential diagnoses. A teaching course for health workers, a picture atlas and guidelines for management have been developed (WHO, 2012; WHO, 2013; WHO, 2017).

Treatment with anti-leishmanial drugs should be with extremely safe and effective drugs as PKDL usually causes limited morbidity without mortality. Regimens should be short course, oral administration allowing treatment on an out-patient basis. Group precision is needed as PKDL patients, in contrast to $\mathrm{VL}$, are not ill and are not malnourished and PK/PD data are required. In addition, drug levels in the skin need to be studied vis-a-vis systemic drug levels, for each drug used, including intrinsic immune effects. This is currently being studied in drug trials in East-Africa and the ISC.

In the ISC all patients are treated with miltefosine for 3 months. A particular problem occurs in the evaluation of cure in macular PKDL as the repigmentation may take a long time and continues after parasitological and immunological cure (Verma et al., 2015; Zijlstra, 2016; Moulik et al., 2018). qPCR was useful to monitor treatment response in patients treated with miltefosine who had a good clinical and parasitological response while in patients treated with AmBisome the response was unsatisfactory as measured by qPCR (Moulik et al., 2018). Miltefosine resistance has been reported (Ramesh et al., 2015).

A new treatment modality to be explored is the use of biologicals. Blockade of PD-1 or PD-1 ligand may promote parasite clearance and increased pro-inflammatory cytokine production as was shown in experimental VL. In PKDL in the ISC, the presence of PD- 1 was demonstrated in dermal lesions by increased mRNA expression of $\mathrm{PD}-1$ and by immunohistochemistry, and in the peripheral blood, while this decreased after treatment and was not demonstrated in healthy controls (Mukherjee et al., 2019). From a clinical point of view, an immune block seems to exist in PKDL in the ISC with no tendency to self-heal, in contrast to the experience in Africa. The use of biologicals in PKDL (and VL) warrants further investigation, for efficacy and safety, and cost-effectiveness.

Biomarkers would include cytokine ratios that could be used as a better, earlier and more direct reflection of the pathophysiology compared to parasitological tools; for example the ratio of a pro-inflammatory cytokine such TNF- $\alpha$ and IL-1 vs. an anti-inflammatory cytokine such as IL-10 and TGF- $\beta$ could be measured before during and after treatment, as a sole cytokines or a part of a cytokine panel. The use of 3-dimensional scanning may be also explored further in diagnosis and as a biomarker in each of the main types (macular and papulonodular). These two modalities should provide precision at the regional level as the differential diagnosis may differ.

Immunochemotherapy may be second line treatment in chronic or refractory cases; the ChAd63-KH vaccine seems a suitable candidate to further build on previous experience with the autoclaved L. major vaccine (Younis et al., 2021).

\subsubsection{Prevention}

Prevention is of paramount importance and seems within reach. Given the increasing understanding of the immune responses, immunological manipulation is attractive to prevent PKDL, and may be combined with drug treatment for VL to enhance or accelerate the immune response, leading to definite cure with reduced risk of VL relapse and development of PKDL. Given the differences in epidemiology and pathophysiology PKDL, more (intra-)regional precision is needed.

Infectivity of PKDL to sand flies should have priority as this is likely to be the driving force in transmission. A complete understanding is needed as to which patients contribute most and at which stage of PKDL, both in terms of numbers (quantity) as in terms of quality (macular vs. polymorphic; early vs. late, limited vs. generalized, etc.). While major progress has been made in infectivity studies from Bangladesh and India, no conclusive studies data exist from East Africa that have used modern tools such as PCR in large numbers of patients (Mondal et al., 2018).

\subsubsection{Asymptomatic Infection}

Current pitfalls in asymptomatic infection include definition, pathophysiology, regional differences, progress to VL and infectivity (Zijlstra et al., 1994; Saha et al., 2017; Sundar and Singh, 2018; Das et al., 2020; Singh et al., 2021).

\subsubsection{Definition}

After infection by a sand fly bite, not all individuals develop clinical VL. Theoretically, three groups may be identified: those who do not have any sign of infection; these remain undetected. The second group are those in whom (conversion in) a positive serological or immunological test is found, but are healthy; these are called asymptomatics. While most individuals remain asymptomatic, a small number develop clinical disease over time.

The third group develop clinical VL, as demonstrated by the presence of parasites or another diagnostic test.

There is no uniform definition of asymptomatic infection. Most studies would advocate the use of a serological test such as the DAT or rK39 ELISA; in longitudinal studies conversion from negative to a positive test would provide strong evidence of a recent infection. Other studies use the LST as an additional marker; here the presence of (conversion in) the LST would also provide evidence for infection leading to immunity. In HIV infected patients infected with $L$. infantum, cytokine release assays were shown to be useful tools (Ibarra-Meneses et al., 2017; Botana et al., 2019). The ratio of clinical VL to 
asymptomatic to varies from 1:9 in India and Nepal, while in Bangladesh this was 1:4. In contrast, in a study in Sudan, in the very beginning of an outbreak after a long disease free interval, clinical infection outnumbered asymptomatic infection by 2.4:1, suggesting low herd immunity (Zijlstra et al., 1994; Bern and Chowdhury, 2006; Ostyn et al., 2011).

There is a need for a more precise definition of asymptomatic infection in each noso-geographic region to understand its importance in transmission. This would take into account factors relating to the host, parasite and sand fly. Studies using molecular tools, immune markers and genetic studies are needed to further describe the importance of asymptomatic infection in terms accurately detection of infection, the risk of developing VL and the risk of transmission of progression to clinical VL, and the role in transmission.

\subsubsection{Pathophysiology}

Clustering in households is well described and may be due transmission related factors such as the presence of VL or PKDL cases or the presence of sand flies and a potential animal reservoir. It is not certain if genetic predisposition in families may play a role (Bern et al., 2010).

It should be noted that the degree of immunity in asymptomatic infection cannot be quantified and this could vary over-time in the natural history, in the course of a concurrent infection, with age, in the course of an outbreak, or during on-going endemic transmission. For example, 32 of 42 asymptomatic individuals cleared the infection as demonstrated by qPCR within 6 months (Das et al., 2020).

\subsubsection{Progression to $V L$}

The importance of asymptomatic infection lies in the presence of leishmania parasites and the possible progression to clinical VL; obviously these individuals need treatment. Markers that have been proposed include high antibody titres in serological tests (DAT, rK39 ELISA), ADA, IL-10, qPCR and LAMP (Chakravarty et al., 2019; Das et al., 2020).

Progression to VL is a considerable risk in HIV co-infection and a screen-and-treat strategy has been developed (van Griensven et al., 2014).

It may be useful to describe the developing immune response in terms of pro- and anti-inflammatory cytokine ratios such as between TNF- $\alpha$ or IL-10, and IFN- $\gamma$. Alternatively, measurement of adenosine deaminase (ADA) as an aspecific immune marker present in all tissues (isoenzyme ADA-1) or in monocytes macrophages (ADA-2) was suggested as a better marker than IL-10 to predict progression to VL (Vijayamahantesh et al., 2016).

Follow-up is essential in asymptomatic patients who are labelled on the basis of as having a positive serological test. It is important to detect those who progress to clinical VL; here molecular tools such as $\mathrm{qPCR}$ have proved indispensable. In a recent study in India, combined rK39 and DAT were find optimal to identify early asymptomatic infection; combining qPCR with serology detected $23.8 \%$ of asymptomatic cases that converted to clinical VL (Das et al., 2020). The clustering in households may be explored in association to genetic markers that have been linked to asymptomatic infection such as alleles of HLA-DR $\beta$ (Chakravarty et al., 2019).

\subsubsection{Infectivity}

While for the asymptomatic individual progression to $\mathrm{VL}$ is obviously important, for the contribution of asymptomatics to transmission, xenodiagnostic studies are pivotal (Singh et al., 2020). For control efforts, the infectiousness of PKDL and asymptomatics as the main contenders, should be quantified in such experiments and balanced against their respective incidence.

Xenodiagnostic studies have been done in PKDL, and are ongoing in asymptomatics in Bangladesh and India (Molina et al., 2017). A recent report from India did not provide support for a significant role of asymptomatics in transmission (Singh et al., 2021). In East Africa, xenodiagnostic studies have shown that $3.2 \%$ of the most infected people were responsible for infection of about $65 \%$ of sand fly vector population, and this needs to be tailored to who contributes most (Miller et al., 2014). Similar studies focusing on asymptomatic infection are needed urgently.

\subsubsection{Transmission and Infection}

Current pitfalls in transmission and infection include factors relating to the sand fly vector, the parasite and the host. Characteristics and determinants of sand fly transmission have been described for each region; a full description is beyond the scope of this paper (Bern et al., 2010; Elnaiem, 2011; Miller et al., 2014; Aklilu et al., 2017).

The epidemiology of sand fly distribution and habitat and contribution to transmission needs regional precision as to spatial and seasonal fluctuations, and the firm identification of the reservoir.

Sand fly competence may be influenced by genetic exchange with leishmania parasites and co-infection; infection with other phleboviruses or other microbiota may be important and could be targeted for intervention. Sand fly saliva has among other, immunomodulatory properties and multiple bites may result in increasing degree of immunization in the naive host with implications for parasite load in the host and clinical implications including asymptomatic infection or clinical VL (Lestinova et al., 2017). Vaccination studies could explore the option of a combined vector salivary protein with a Leishmania antigen (Kamhawi et al., 2014).

Both in the ISC and in East Africa there is no consensus on the existence of an animal reservoir. While leishmania parasites have been demonstrated in animals, no evidence exist that these animals contribute to transmission (Bourdeau et al., 2020; Kushwaha et al., 2021).

Xenodiagnostic studies may be considered the gold standard for determining infectiousness. However, standardization of protocols is needed. Interpretation of findings should take into account that xenodiagnosis may not necessarily mimic the natural situation as sand fly competency in the wild may be different from what is found in the lab. Other variables that are difficult to control are parts of the body exposed to sand fly bites and the natural feeding timing of sand flies during the day (Singh et al., 2020). While studies in transmission including xenodiagnosis on PKDL and asymptomatic infection are well 
TABLE 1 | Priorities for precision in VL and PKDL research.

Diagnosis

Treatment and immune manipulation

\section{Genomic tools}

O Metagenomics - identification of co-infection

Transcriptomics - genetic determinants ('gene signature') for diagnosis and outcome

O Nanodiagnostics - superior accuracy

Adapted tools

Use of multiplex PCR in differential diagnosis

O Use of panel of cytokine markers in diagnosis and as a biomarker.

Re-development of the LST that is safe and antigenic in all endemic areas

Priorities - general

Development of PCR based diagnosis under field conditions

Explore combination of tests, e.g. an anti- and a pro-inflammatory marker.

Evaluation of diagnostic tests in longitudinal studies rather than in stored samples to assess PPV and NPV

Explore paired strains of VL and PKDL patients Vs. VL strains of patients who do not develop PKDL

Priorities - biomarker

Focus on immune parameters that reflect cell-mediated immunity, e.g. a cytokine ratio

Relationship between parasite detection tests (PCR, antigen tests) and immune responses during and after treatment

Serological tests are unlikely to be useful as a biomarker as a sole test

Treatment target

Shift of focus from parasitological killing to assessment of drug- induced protective immune responses including those mediated by the drug

Examine the benefit and risk of non-sterile cure vs sterile cure, at individual and population level

Evaluation of treatment

Evaluate treatment efficacy in relation to parasite load, co-infection and malnutrition, for individual precision

Evaluate treatment efficacy and safety in all populations including (pregnant) women and children

Develop drugs in parallel with biomarker (theragnostics)

- Explore population pharmacokinetic modelling for anti-leishmania drugs in each endemic area

Describe genetic factors (host and parasite derived) that predict efficacy and/or toxicity of anti-leishmania drugs in endemic regions

O PK/PD studies in all drug studies including penetration in the skin as a parameter to prevent and /or treat PKDL

Nanotechnology to detect drug resistance

Immune manipulation

Evaluation of immunomodulators

Prophylactic vaccination for VL; prophylactic and therapeutic vaccination for PKDL

Cost-effectiveness of interventions

Use of pharmaco-economics to assess cost efficiency (e.g. drug treatment vs. vaccination)

PKDL

Priorities in diagnosis and biomarker

Use of multiplex PCR in differential diagnosis

Explore the use of artificial intelligence (deep learning) to recognize and (differential) diagnose at field level

Explore immunological parameters, e.g. a ratio of a anti- and pro-inflammatory cytokine

Explore 3 -dimensional scanning as a biomarker

Priorities in treatment

S Short, ambulatory, safe and effective treatment with aim of pushing the immune response towards a cure profile

$\mathrm{O}$ Explore differences in PK/PD in treatment of PKDL vs treatment of VL, including drug levels in the skin

Explore use of biologicals

Explore use of immune modulator or prophylactic/ therapeutic vaccine

Priorities in prevention

Optimal drug treatment for $\mathrm{VL}$ with lowest possible PKDL rate

Explore pathophysiological trigger for late occurrence of PKDL in the ISC - intercurrent infection (helminths), loss of immunological memory (as in measles), other factors

O Prophylactic vaccine to be used in combination with VL treatment

Definition

Describe uniform definition

O Describe determinants (host, parasite, vector)

$\mathrm{D}$ Define robust markers for progression to $\mathrm{VL}$ that can be used in the field

Epidemiology

Description (incidence), characterization and infectivity in early and late phase of outbreaks and in endemic transmission

Determine infectivity in all endemic areas Infectivity

Establish uniform protocols (PCR, sand fly bites)

Describe infectivity in the whole spectrum of $\mathrm{VL}$ and PKDL, including HIV co-infection

Describe changes in infectivity in the ISC after the start of treatment

Determine infectivity in Africa in papular /nodular and macular PKDL, in early vs late development of PKDL, in acute vs chronic PKDL, according to age group

Transmission

Examine a possible animal reservoir in both East Africa and the ISC

F Further develop model for monitoring in ISC

O Develop model for interventions in Africa 
under way in the ISC, East Africa lags far behind; also here there is a need for regional precision.

Modelling is a powerful tool in precision medicine and global health, and key in decision making in control programs, such as the KAEP. There is no modelling effort for East Africa. Parameters of the model may relate to infection by sand flies (exposure, indoor or outdoor transmission, house-hold transmission, seasonality), outcome of infection (active VL, exVL, PKDL [macular vs polymorphic, interval after VL, self-cure or not], asymptomatic infection, VL-HIV co-infection), and infectivity of infected individuals to sand flies. This includes the presence of an animal reservoir. Issues on (life-long) acquired immunity, the effect on persisting parasites and transmissibility are important determinants. It follows that all parameters should be described in as much detail as possible as to their contribution. Also here regional precision is needed to be able to target interventions. These may focus on relatively few superspreaders such as VL patients or HIV-VL co-infected patients during epidemics, while during inter-epidemic periods asymptomatic (high numbers, probably low infectivity) and/or PKDL patients (low numbers, high infectivity) may play a role in new outbreaks. Clearly these efforts should take into account the introduction of a vaccine, active or passive case detection, sand fly control by insecticide residual spraying, bed nets, or combinations, etc. (Chapman et al., 2018a; Chapman et al., 2018b; Le Rutte et al., 2019)

\section{SUMMARY}

Precision medicine and global health offer the possibility to examine and to apply genomic and non-genomic tools in control of VL caused by L. donovani, with focus on the five priorities (diagnosis, treatment, PKDL, asymptomatic infection and transmission), all of which are interlinked by the immune responses. These new and powerful tools have contributed considerably to our understanding of the pathophysiology and epidemiology of VL and have the potential to change and revolutionize our approach in research and control. (Table 1).

Genomics offers new tools in diagnosis based on the interaction between parasite, host and vector, of which transcriptomics seems most promising. While these tools still need to be developed and evaluated further for applicability in an LMIC setting, they have the potential to replace traditional tools such as serological diagnosis with improved accuracy, while at the same time providing information on protective immune response. Similar advantages may be expected in treatment when the immune response is linked to the genomics in endemic populations, such as transcriptomic profiles. The increased understanding of protective immune responses may very well lead in the long term to priority of vaccine development in relation to drug development, while combinations such as immunochemotherapy may be of medium term benefit. In addition, non-genomic tools (e.g. the LST) or further development of existing (molecular) tools (e.g. multiplex platforms for PCR and cytokines) offer rapid assessment of the immune responses.
Description of genomic and non-genomic tools is relevant to PKDL and asymptomatic infection to describe risk factors with relation to host, parasite and vector. Here the understanding of immune responses is key, including potential triggers such as intercurrent infection, or factors relevant for protection (in VL to develop PKDL) or progression VL clinical disease (in asymptomatics). In transmission, the genetic exchange between vector and parasites or symbionts and effect of vector saliva on host immune responses when exposed is likely to influence vaccine development.

Key in both VL and PKDL is the understanding and manipulation of the (developing) immune response that is determined by, among other, genetic factors in the host, the parasite and the sand fly. (Figure 1) The immune response acts as a major final common pathway in the 5 priorities (Table 1). In this respect, a change in paradigm may be needed from classical microscopical diagnosis and parasite-killing drugs, to immunebased diagnosis and biomarkers, drugs in combination with immune modulation, and vaccination.

\section{CONCLUSION}

Research efforts in leishmaniasis are often fragmented, vertical and not well coordinated; for example, development of drugs, diagnostic tools, or vaccines are often done independently. Studies on basic science, pathophysiology and immune responses often do not run in parallel with clinical research. The lack of a holistic approach causes inefficiency, unnecessary delays and inefficient use of funds.

There is a need to continually explore the landscape, and include and use innovative approaches that arise in the whole field of internal medicine and paediatrics, radiology, laboratory and basic science, etc. Studies on cross-cutting issues with other tropical conditions could lead to better efficiency e.g. the study of asymptomatics and immune responses among the kinetoplastic diseases (human African trypanosomiasis and Chagas' disease). Genomic tools have the potential to replace traditional tools in pathophysiology, management and control, offering precision in each endemic area. Precision in VL (in clinical medicine as well as public health) should currently be tailored to the (intra-) regional level given the heterogeneity of hosts, parasites and sand flies. Tailored treatment is already the case in HIV-VL coinfected patients, and should apply to management of VL and PKDL in the future. The next step would be to strengthen the ongoing research efforts for precision for groups (e.g. children, women, pregnancy, VL-HIV co-infection). Once this has been achieved, further development for precision at the individual level will not be unrealistic.

\section{AUTHOR CONTRIBUTIONS}

The author confirms being the sole contributor of this work and has approved it for publication. 


\section{REFERENCES}

Abeijon, C., Alves, F., Monnerat, S., Mbui, J., Viana, A. G., Almeida, R. M., et al. (2020). Urine-Based Antigen Detection Assay for Diagnosis of Visceral Leishmaniasis Using Monoclonal Antibodies Specific for Six Protein Biomarkers of Leishmania Infantum/Leishmania Donovani. PloS Negl. Trop. Dis. 14 (4), e0008246. doi: 10.1371/journal.pntd.0008246

Adaui, V., Lye, L. F., Akopyants, N. S., Zimic, M., Llanos-Cuentas, A., Garcia, L., et al. (2016). Association of the Endobiont Double-Stranded RNA Virus LRV1 With Treatment Failure for Human Leishmaniasis Caused by Leishmania Braziliensis in Peru and Bolivia. J. Infect. Dis. 213 (1), 112-121. doi: 10.1093/ infdis/jiv354

Adriaensen, W., Cuypers, B., Cordero, C. F., Mengasha, B., Blesson, S., Cnops, L., et al. (2020). Host Transcriptomic Signature as Alternative Test-of-Cure in Visceral Leishmaniasis Patients Co-Infected With HIV. EBioMedicine 55, 102748. doi: 10.1016/j.ebiom.2020.102748

Aklilu, E., Gebresilassie, A., Yared, S., Kindu, M., Tekie, H., Balkew, M., et al. (2017). Studies on Sand Fly Fauna and Ecological Analysis of Phlebotomus Orientalis in the Highland and Lowland Foci of Kala-Azar in Northwestern Ethiopia. PloS One 12 (4), e0175308. doi: 10.1371/journal.pone.0175308

Akopyants, N. S., Kimblin, N., Secundino, N., Patrick, R., Peters, N., Lawyer, P., et al. (2009). Demonstration of Genetic Exchange During Cyclical Development of Leishmania in the Sand Fly Vector. Science 324 (5924), 265-268. doi: 10.1126/science.1169464

Akuffo, H., Costa, C., van Griensven, J., Burza, S., Moreno, J., and Herrero, M. (2018). New Insights Into Leishmaniasis in the Immunosuppressed. PloS Negl. Trop. Dis. 12 (5), e0006375. doi: 10.1371/journal.pntd.0006375

Alkhaldy, H. Y., Badri, R., and Eldeen Bakheet, O. S. (2020). Visceral Leishmaniasis With Hemophagocytic Lymphohistiocytosis (HLH). IDCases 21, e00804. doi: 10.1016/j.idcr.2020.e00804

Alvar, J., Velez, I. D., Bern, C., Herrero, M., Desjeux, P., Cano, J., et al. (2012). Leishmaniasis Worldwide and Global Estimates of its Incidence. PloS One 7 (5), e35671. doi: 10.1371/journal.pone.0035671

Alves, F., Bilbe, G., Blesson, S., Goyal, V., Monnerat, S., Mowbray, C., et al. (2018). Recent Development of Visceral Leishmaniasis Treatments: Successes, Pitfalls, and Perspectives. Clin. Microbiol. Rev. 31 (4). doi: 10.1128/CMR.00048-18

Ansari, N. A., Katara, G. K., Ramesh, V., and Salotra, P. (2008). Evidence for Involvement of TNFR1 and TIMPs in Pathogenesis of Post-Kala-Azar Dermal Leishmaniasis. Clin. Exp. Immunol. 154 (3), 391-398. doi: 10.1111/j.13652249.2008.03761.x

Ansari, N. A., Ramesh, V., and Salotra, P. (2008). Immune Response Following Miltefosine Therapy in a Patient With Post-Kala-Azar Dermal Leishmaniasis. Trans. R. Soc. Trop. Med. Hyg. 102 (11), 1160-1162. doi: 10.1016/ j.trstmh.2008.05.015

Antinori, S., Calattini, S., Longhi, E., Bestetti, G., Piolini, R., Magni, C., et al. (2007). Clinical Use of Polymerase Chain Reaction Performed on Peripheral Blood and Bone Marrow Samples for the Diagnosis and Monitoring of Visceral Leishmaniasis in HIV-Infected and HIV-Uninfected Patients: A Single-Center, 8-Year Experience in Italy and Review of the Literature. Clin. Infect. Dis.: Off. Publ. Infect. Dis. Soc. America 44 (12), 1602-1610. doi: 10.1086/518167

Baker, J. L., Shriner, D., Bentley, A. R., and Rotimi, C. N. (2017). Pharmacogenomic Implications of the Evolutionary History of Infectious Diseases in Africa. Pharmacogenomics J. 17 (2), 112-120. doi: 10.1038/ tpj.2016.78

Bermudi, P. M. M., Costa, D., Nunes, C. M., Tolezano, J. E., Hiramoto, R. M., Rodas, L. A. C., et al. (2020). Canine Serological Survey and Dog Culling Ant its Relationship With Human Visceral Leishmaniasis in an Endemic Urban Area. BMC Infect. Dis. 20 (1), 401. doi: 10.1186/s12879-020-05125-0

Bern, C., Amann, J., Haque, R., Chowdhury, R., Ali, M., Kurkjian, K. M., et al. (2006). Loss of Leishmanin Skin Test Antigen Sensitivity and Potency in a Longitudinal Study of Visceral Leishmaniasis in Bangladesh. Am. J. Trop. Med. Hyg. 75 (4), 744-748. doi: 10.4269/ajtmh.2006.75.744

Bern, C., and Chowdhury, R. (2006). The Epidemiology of Visceral Leishmaniasis in Bangladesh: Prospects for Improved Control. Indian J. Med. Res. 123 (3), 275-288.

Bern, C., Courtenay, O., and Alvar, J. (2010). Of Cattle, Sand Flies and Men: A Systematic Review of Risk Factor Analyses for South Asian Visceral
Leishmaniasis and Implications for Elimination. PloS Negl. Trop. Dis. 4 (2), e599. doi: 10.1371/annotation/f96dd2ce-05de-4aa5-a9d5-c481b0745c84

Blackwell, J. M., Fakiola, M., and Castellucci, L. C. (2020). Human Genetics of Leishmania Infections. Hum. Genet. 139 (6-7), 813-819. doi: 10.1007/s00439020-02130-w

Blackwell, J. M., Fakiola, M., and Singh, O. P. (2020). Genetics, Transcriptomics and Meta-Taxonomics in Visceral Leishmaniasis. Front. Cell. Infect. Microbiol. 10, 590888. doi: 10.3389/fcimb.2020.590888

Boelaert, M., Verdonck, K., Menten, J., Sunyoto, T., van Griensven, J., Chappuis, F., et al. (2014). Rapid Tests for the Diagnosis of Visceral Leishmaniasis in Patients With Suspected Disease. Cochrane Database Syst. Rev. 6, CD009135. doi: 10.1002/14651858.CD009135.pub2

Bolia, R., Singh, A., Maji, M., Misra, M., Sharawat, I. K., and Panda, P. K. (2020). Visceral Leishmaniasis Associated With Hemophagocytic Lymphohistiocytosis. Indian J. Pediatr. 88(1), 73. doi: 10.1007/s12098-02003361-5

Botana, L., Ibarra-Meneses, A. V., Sanchez, C., Castro, A., San Martin, J. V., Molina, L., et al. (2019). Asymptomatic Immune Responders to Leishmania Among HIV Positive Patients. PloS Negl. Trop. Dis. 13 (6), e0007461. doi: 10.1371/journal.pntd.0007461

Bourdeau, P., Rowton, E., and Petersen, C. (2020). Impact of Different Leishmania Reservoirs on Sand Fly Transmission: Perspectives From Xenodiagnosis and Other One Health Observations. Vet. Parasitol. 287, 109237. doi: 10.1016/ j.vetpar.2020.109237

Brodskyn, C. I., and Kamhawi, S. (2018). Biomarkers for Zoonotic Visceral Leishmaniasis in Latin America. Front. Cell. Infect. Microbiol. 8, 245. doi: $10.3389 /$ fcimb. 2018.00245

Campolina, T. B., Villegas, L. E. M., Monteiro, C. C., Pimenta, P. F. P., and Secundino, N. F. C. (2020). Tripartite Interactions: Leishmania, Microbiota and Lutzomyia Longipalpis. PloS Negl. Trop. Dis. 14 (10), e0008666. doi: 10.1371/journal.pntd.0008666

Carnielli, J. B. T., Crouch, K., Forrester, S., Silva, V. C., Carvalho, S. F. G. Damasceno, J. D., et al. (2018). A Leishmania Infantum Genetic Marker Associated With Miltefosine Treatment Failure for Visceral Leishmaniasis. EBioMedicine 36, 83-91. doi: 10.1016/j.ebiom.2018.09.029

Carnielli, J. B. T., Monti-Rocha, R., Costa, D. L., Molina Sesana, A., Pansini, L. N. N., Segatto, M., et al. (2019). Natural Resistance of Leishmania Infantum to Miltefosine Contributes to the Low Efficacy in the Treatment of Visceral Leishmaniasis in Brazil. Am. J. Trop. Med. Hyg. 101 (4), 789-794. doi: 10.4269/ ajtmh.18-0949

Chakravarty, J., Hasker, E., Kansal, S., Singh, O. P., Malaviya, P., Singh, A. K., et al. (2019). Determinants for Progression From Asymptomatic Infection to Symptomatic Visceral Leishmaniasis: A Cohort Study. PloS Negl. Trop. Dis. 13 (3), e0007216. doi: 10.1371/journal.pntd.0007216

Chapman, L. A. C., Jewell, C. P., Spencer, S. E. F., Pellis, L., Datta, S., Chowdhury, R., et al. (2018a). The Role of Case Proximity in Transmission of Visceral Leishmaniasis in a Highly Endemic Village in Bangladesh. PloS Negl. Trop. Dis. 12 (10), e0006453. doi: 10.1371/journal.pntd.0006453

Chapman, L. A. C., Morgan, A. L. K., Adams, E. R., Bern, C., Medley, G. F., and Hollingsworth, T. D. (2018b). Age Trends in Asymptomatic and Symptomatic Leishmania Donovani Infection in the Indian Subcontinent: A Review and Analysis of Data From Diagnostic and Epidemiological Studies. PloS Negl. Trop. Dis. 12 (12), e0006803. doi: 10.1371/journal.pntd.0006803

Chaudhry, M., Alessandrini, M., and Pepper, M. S. (2016). Pharmacogenomics for Infectious Diseases in Sub-Saharan Africa: Successes and Opportunities. Appl. Transl. Genom 9, 3-5. doi: 10.1016/j.atg.2016.04.001

Cloots, K., Burza, S., Malaviya, P., Hasker, E., Kansal, S., Mollett, G., et al. (2020). Male Predominance in Reported Visceral Leishmaniasis Cases: Nature or Nurture? A Comparison of Population-Based With Health Facility-Reported Data. PloS Negl. Trop. Dis. 14 (1), e0007995. doi: 10.1371/journal.pntd.0007995

Cloots, K., Singh, O. P., Singh, A. K., van der Auwera, G., Kumar, P., Gedda, M. R., et al. (2021). Assessing L. Donovani Skin Parasite Load: A Proof of Concept Study of a Microbiopsy Device in an Indian Setting. Front. Cell. Infect. Microbiol. 11, 645121. doi: 10.3389/fcimb.2021.645121

Colomba, C., Saporito, L., Di Carlo, P., Tolomeo, M., Cervo, A., Firenze, A., et al. (2019). Direct-Acting Antivirals and Visceral Leishmaniasis: A Case Report. BMC Infect. Dis. 19 (1), 328. doi: 10.1186/s12879-019-3947-x 
Das, V. N. R., Bimal, S., Siddiqui, N. A., Kumar, A., Pandey, K., Sinha, S. K., et al. (2020). Conversion of Asymptomatic Infection to Symptomatic Visceral Leishmaniasis: A Study of Possible Immunological Markers. PloS Negl. Trop. Dis. 14 (6), e0008272. doi: 10.1371/journal.pntd.0008272

Dedet, J. P., Roche, B., Pratlong, F., Cales-Quist, D., Jouannelle, J., Benichou, J. C., et al. (1995). Diffuse Cutaneous Infection Caused by a Presumed Monoxenous Trypanosomatid in a Patient Infected With HIV. Trans. R. Soc. Trop. Med. Hyg. 89 (6), 644-646. doi: 10.1016/0035-9203(95)90427-1

Dey, R., Joshi, A. B., Oliveira, F., Pereira, L., Guimaraes-Costa, A. B., Serafim, T. D., et al. (2018). Gut Microbes Egested During Bites of Infected Sand Flies Augment Severity of Leishmaniasis via Inflammasome-Derived IL-1beta. Cell Host Microbe 23 (1), 134-43 e6. doi: 10.1016/j.chom.2017.12.002

Dey, A., and Singh, S. (2007). Genetic Heterogeneity Among Visceral and PostKala-Azar Dermal Leishmaniasis Strains From Eastern India. Infect. Genet. Evol.: J. Mol. Epidemiol. Evol. Genet. Infect. Dis. 7 (2), 219-222. doi: 10.1016/ j.meegid.2006.09.001

Diro, E., Blesson, S., Edwards, T., Ritmeijer, K., Fikre, H., Admassu, H., et al. (2019). A Randomized Trial of AmBisome Monotherapy and AmBisome and Miltefosine Combination to Treat Visceral Leishmaniasis in HIV Co-Infected Patients in Ethiopia. PloS Negl. Trop. Dis. 13 (1), e0006988. doi: 10.1371/ journal.pntd.0006988

Diro, E., Lynen, L., Ritmeijer, K., Boelaert, M., Hailu, A., and van Griensven, J. (2014). Visceral Leishmaniasis and HIV Coinfection in East Africa. PloS Negl. Trop. Dis. 8 (6), e2869. doi: 10.1371/journal.pntd.0002869

Ditgen, D., Anandarajah, E. M., Meissner, K. A., Brattig, N., Wrenger, C., and Liebau, E. (2014). Harnessing the Helminth Secretome for Therapeutic Immunomodulators. BioMed. Res. Int. 2014, 964350. doi: 10.1155/2014/964350

El Hassan, A. M., Khalil, E. A., Elamin, W. M., El Hassan, L. A., Ahmed, M. E., and Musa, A. M. (2013). Misdiagnosis and Mistreatment of Post-Kala-Azar Dermal Leishmaniasis. Case Rep. Med. 2013, 351579. doi: 10.1155/2013/351579

Elnaiem, D. E. (2011). Ecology and Control of the Sand Fly Vectors of Leishmania Donovani in East Africa, With Special Emphasis on Phlebotomus Orientalis. J. Vector Ecol.: J. Soc. Vector Ecol. 36 Suppl 1, S23-S31. doi: 10.1111/j.19487134.2011.00109.x

Fakiola, M., Singh, O. P., Syn, G., Singh, T., Singh, B., Chakravarty, J., et al. (2019). Transcriptional Blood Signatures for Active and Amphotericin B Treated Visceral Leishmaniasis in India. PloS Negl. Trop. Dis. 13 (8), e0007673. doi: 10.1371/journal.pntd.0007673

Fakiola, M., Strange, A., Cordell, H. J., Miller, E. N., Pirinen, M., Su, Z., et al. (2013). Common Variants in the HLA-DRB1-HLA-DQA1 HLA Class II Region Are Associated With Susceptibility to Visceral Leishmaniasis. Nat. Genet. 45 (2), 208-213. doi: 10.1038/ng.2518

Faleiro, R. J., Kumar, R., Hafner, L. M., and Engwerda, C. R. (2014). Immune Regulation During Chronic Visceral Leishmaniasis. PloS Negl. Trop. Dis. 8 (7), e2914. doi: 10.1371/journal.pntd.0002914

Fararouei, M., Sarkari, B., Abdolahi Khabisi, S., and Rezaei, Z. (2018). Diagnostic Accuracy of Urinary Latex Agglutination Test (KAtex) for the Diagnosis of Visceral Leishmaniasis: A Meta-Analysis. J. Infect. Dev. Ctries. 12 (12), 10451051. doi: $10.3855 /$ jidc. 10185

Farouk, S., Salih, M. A., Musa, A. M., Blackwell, J. M., Miller, E. N., Khalil, E. A., et al. (2010). Interleukin 10 Gene Polymorphisms and Development of Post Kala-Azar Dermal Leishmaniasis in a Selected Sudanese Population. Public Health Genomics 13 (6), 362-367. doi: 10.1159/000272457

Flahault, A., Geissbuhler, A., Guessous, I., Guerin, P. J., Bolon, I., Salathe, M., et al. (2017). Precision Global Health in the Digital Age. Swiss Med. Wkly. 147, w14423. doi: 10.4414/smw.2017.14423

Flahault, A., and Salathe, M. (2017). Using Big Data for Disease Surveillance and Drug Safety Monitoring. La Rev. Du Praticien 67 (1), 25-30.

Gedda, M. R., Madhukar, P., Shukla, A., Mudavath, S. L., Srivastava, O. N., Singh, O. P., et al. (2021). Nanodiagnostics in Leishmaniasis: A New Frontiers for Early Elimination. Wiley Interdiscip. Rev. Nanomed. Nanobiotechnol. 13 (2), e1675. doi: 10.1002/wnan. 1675

Ghosh, S., Banerjee, P., Sarkar, A., Datta, S., and Chatterjee, M. (2012). Coinfection of Leptomonas Seymouri and Leishmania Donovani in Indian Leishmaniasis. J. Clin. Microbiol. 50 (8), 2774-2778. doi: 10.1128/JCM.00966-12

Ghosh, M., Roy, K., and Roy, S. (2013). Immunomodulatory Effects of Antileishmanial Drugs. J. Antimicrobial. Chemother. 68 (12), 2834-2838. doi: $10.1093 / \mathrm{jac} / \mathrm{dkt} 262$
Ginouves, M., Couppie, P., Simon, S., Bourreau, E., Rogier, S., Brousse, P., et al. (2021). Leishmaniavirus Genetic Diversity Is Not Related to Leishmaniasis Treatment Failure. Clin. Microbiol. Infect.: Off. Publ. Eur. Soc. Clin. Microbiol. Infect. Dis. 27 (2), 286 e1-286.e5. doi: 10.1016/j.cmi.2020.04.037

Goyal, V., Das, V. N. R., Singh, S. N., Singh, R. S., Pandey, K., Verma, N., et al. (2020). Long-Term Incidence of Relapse and Post-Kala-Azar Dermal Leishmaniasis After Three Different Visceral Leishmaniasis Treatment Regimens in Bihar, India. PloS Negl. Trop. Dis. 14 (7), e0008429. doi: 10.1371/journal.pntd.0008429

Gramiccia, M., Bettini, S., Gradoni, L., Ciarmoli, P., Verrilli, M. L., Loddo, S., et al. (1990). Leishmaniasis in Sardinia. 5. Leishmanin Reaction in the Human Population of a Focus of Low Endemicity of Canine Leishmaniasis. Trans. $R$. Soc. Trop. Med. Hyg. 84 (3), 371-374. doi: 10.1016/0035-9203(90)90322-6

Griffin, D. E. (2010). Measles Virus-Induced Suppression of Immune Responses. Immunol. Rev. 236, 176-189. doi: 10.1111/j.1600-065X.2010.00925.x

Grybchuk, D., Macedo, D. H., Kleschenko, Y., Kraeva, N., Lukashev, A. N., Bates, P. A., et al. (2020). The First Non-LRV RNA Virus in Leishmania. Viruses 12 (2), 168. doi: $10.3390 / \mathrm{v} 12020168$

Hailu, A., Musa, A., Wasunna, M., Balasegaram, M., Yifru, S., Mengistu, G., et al. (2010). Geographical Variation in the Response of Visceral Leishmaniasis to Paromomycin in East Africa: A Multicentre, Open-Label, Randomized Trial. PloS Negl. Trop. Dis. 4 (10), e709. doi: 10.1371/journal.pntd.0000709

Ibarra-Meneses, A. V., Mondal, D., Alvar, J., Moreno, J., and Carrillo, E. (2017). Cytokines and Chemokines Measured in Dried SLA-Stimulated Whole Blood Spots for Asymptomatic Leishmania Infantum and Leishmania Donovani Infection. Sci. Rep. 7 (1), 17266. doi: 10.1038/s41598-017-17315-Z

Jaber, H. T., Hailu, A., Pratlong, F., Lami, P., Bastien, P., and Jaffe, C. L. (2018). Analysis of Genetic Polymorphisms and Tropism in East African Leishmania Donovani by Amplified Fragment Length Polymorphism and kDNA Minicircle Sequencing. Infect. Genet. Evol.: J. Mol. Epidemiol. Evol. Genet. Infect. Dis. 65, 80-90. doi: 10.1016/j.meegid.2018.07.016

Kamhawi, S., Aslan, H., and Valenzuela, J. G. (2014). Vector Saliva in Vaccines for Visceral Leishmaniasis: A Brief Encounter of High Consequence? Front. Public Health 2, 99. doi: 10.3389/fpubh.2014.00099

Karmakar, S., Nath, S., Sarkar, B., Chakraborty, S., Paul, S., Karan, M., et al. (2021). Insect Vectors' Saliva and Gut Microbiota as a Blessing in Disguise: Probability Versus Possibility. Future Microbiol. 16, 657-670. doi: 10.2217/fmb-2020-0239

Khalil, E. A., El Hassan, A. M., Zijlstra, E. E., Mukhtar, M. M., Ghalib, H. W., Musa, B., et al. (2000). Autoclaved Leishmania Major Vaccine for Prevention of Visceral Leishmaniasis: A Randomised, Double-Blind, BCG-Controlled Trial in Sudan. Lancet 356 (9241), 1565-1569. doi: 10.1016/s0140-6736(00)03128-7

Khalil, E. A., Khidir, S. A., Musa, A. M., Musa, B. Y., Elfaki, M. E., Elkadaru, A. M., et al. (2013). Post-Kala-Azar Dermal Leishmaniasis: A Paradigm of Paradoxical Immune Reconstitution Syndrome in Non-HIV/AIDS Patients. J. Trop. Med. 2013, 275253. doi: 10.1155/2013/275253

Khoury, M. J., Iademarco, M. F., and Riley, W. T. (2016). Precision Public Health for the Era of Precision Medicine. Am. J. Prev. Med. 50 (3), 398-401. doi: 10.1016/j.amepre.2015.08.031

Kip, A. E., Blesson, S., Alves, F., Wasunna, M., Kimutai, R., Menza, P., et al. (2021). Low Antileishmanial Drug Exposure in HIV-Positive Visceral Leishmaniasis Patients on Antiretrovirals: An Ethiopian Cohort Study. J. Antimicrobial. Chemother. 76 (5), 1258-1268. doi: 10.1093/jac/dkab013

Kip, A. E., Schellens, J. H. M., Beijnen, J. H., and Dorlo, T. P. C. (2018). Clinical Pharmacokinetics of Systemically Administered Antileishmanial Drugs. Clin. Pharmacokinet. 57 (2), 151-176. doi: 10.1007/s40262-017-0570-0

Koopmans, A. B., Braakman, M. H., Vinkers, D. J., Hoek, H. W., and van Harten, P. N. (2021). Meta-Analysis of Probability Estimates of Worldwide Variation of CYP2D6 and CYP2C19. Transl. Psychiatry 11 (1), 141. doi: 10.1038/s41398020-01129-1

Ko, E. R., Philipson, C. W., Burke, T. W., Cer, R. Z., Bishop-Lilly, K. A., Voegtly, L. J., et al. (2019). Direct-From-Blood RNA Sequencing Identifies the Cause of Post-Bronchoscopy Fever. BMC Infect. Dis. 19 (1), 905. doi: 10.1186/s12879019-4462-9

Kuhls, K., Keilonat, L., Ochsenreither, S., Schaar, M., Schweynoch, C., Presber, W., et al. (2007). Multilocus Microsatellite Typing (MLMT) Reveals Genetically Isolated Populations Between and Within the Main Endemic Regions of Visceral Leishmaniasis. Microbes Infect. Inst. Pasteur 9 (3), 334-343. doi: 10.1016/j.micinf.2006.12.009 
Kumar, R., Chauhan, S. B., Ng, S. S., Sundar, S., and Engwerda, C. R. (2017). Immune Checkpoint Targets for Host-Directed Therapy to Prevent and Treat Leishmaniasis. Front. Immunol. 8, 1492. doi: 10.3389/fimmu.2017.01492

Kumar, R., Pai, K., Pathak, K., and Sundar, S. (2001). Enzyme-Linked Immunosorbent Assay for Recombinant K39 Antigen in Diagnosis and Prognosis of Indian Visceral Leishmaniasis. Clin. Diagn. Lab. Immunol. 8 (6), 1220-1224. doi: 10.1128/CDLI.8.6.1220-1224.2001

Kushwaha, A. K., Scorza, B. M., Singh, O. P., Rowton, E., Lawyer, P., Sundar, S., et al. (2021). Domestic Mammals as Reservoirs for Leishmania Donovani on the Indian Subcontinent: Possibility and Consequences on Elimination. Transbound. Emerg. Dis. 8, 10.1111/tbed.14061. doi: 10.1111/tbed.14061

Le Rutte, E. A., Zijlstra, E. E., and de Vlas, S. J. (2019). Post-Kala-Azar Dermal Leishmaniasis as a Reservoir for Visceral Leishmaniasis Transmission. Trends Parasitol. 35 (8), 590-592. doi: 10.1016/j.pt.2019.06.007

Lestinova, T., Rohousova, I., Sima, M., de Oliveira, C. I., and Volf, P. (2017). Insights Into the Sand Fly Saliva: Blood-Feeding and Immune Interactions Between Sand Flies, Hosts, and Leishmania. PloS Negl. Trop. Dis. 11 (7), e0005600. doi: 10.1371/journal.pntd.0005600

Li, N., Cai, Q., Miao, Q., Song, Z., Fang, Y., and Hu, B. (2021). High-Throughput Metagenomics for Identification of Pathogens in the Clinical Settings. Small Methods 5 (1), 2000792. doi: 10.1002/smtd.202000792

Lima, I. D., Lima, A. L. M., Mendes-Aguiar, C. O., Coutinho, J. F. V., Wilson, M. E., Pearson, R. D., et al. (2018). Changing Demographics of Visceral Leishmaniasis in Northeast Brazil: Lessons for the Future. PloS Negl. Trop. Dis. 12 (3), e0006164. doi: 10.1371/journal.pntd.0006164

Lockard, R. D., Wilson, M. E., and Rodriguez, N. E. (2019). Sex-Related Differences in Immune Response and Symptomatic Manifestations to Infection With Leishmania Species. J. Immunol. Res. 2019, 4103819. doi: $10.1155 / 2019 / 4103819$

Louzir, H., Triki, H., Bennani, S., Edelman, L., Laouini, D., Ben Ismail, R., et al. (1993). Risk Factors for Visceral Leishmaniasis: Role of Epstein-Barr Virus Infection. Arch. Inst. Pasteur Tunis 70 (3-4), 357-362.

Lu, H., Giordano, F., and Ning, Z. (2016). Oxford Nanopore MinION Sequencing and Genome Assembly. Genomics Proteomics Bioinf. 14 (5), 265-279. doi: 10.1016/j.gpb.2016.05.004

Lukes, J., Mauricio, I. L., Schonian, G., Dujardin, J. C., Soteriadou, K., Dedet, J. P., et al. (2007). Evolutionary and Geographical History of the Leishmania Donovani Complex With a Revision of Current Taxonomy. Proc. Natl. Acad. Sci. U. S. A. 104 (22), 9375-9380. doi: 10.1073/pnas.0703678104

Macedo, D. H., Menezes-Neto, A., Rugani, J. M., Rocha, A. C., Silva, S. O., Melo, M. N., et al. (2016). Low Frequency of LRV1 in Leishmania Braziliensis Strains Isolated From Typical and Atypical Lesions in the State of Minas Gerais, Brazil. Mol. Biochem. Parasitol. 210 (1-2), 50-54. doi: 10.1016/j.molbiopara. 2016.08.005

Mandell, M. A., and Beverley, S. M. (2017). Continual Renewal and Replication of Persistent Leishmania Major Parasites in Concomitantly Immune Hosts. Proc. Natl. Acad. Sci. U. S. A. 114 (5), E801-EE10. doi: 10.1073/pnas.1619265114

Martinez, D. Y., Llanos-Cuentas, A., Dujardin, J. C., Polman, K., Adaui, V., Boelaert, M., et al. (2020). A Case-Control Study on the Association Between Intestinal Helminth Infections and Treatment Failure in Patients With Cutaneous Leishmaniasis. Open Forum Infect. Dis. 7 (5), ofaa155. doi: 10.1093/ofid/ofaa155

Matimba, A., Oluka, M. N., Ebeshi, B. U., Sayi, J., Bolaji, O. O., Guantai, A. N., et al. (2008). Establishment of a Biobank and Pharmacogenetics Database of African Populations. Eur. J. Hum. Genet.: EJHG 16 (7), 780-783. doi: 10.1038/ ejhg.2008.49

Mauvais-Jarvis, F., Bairey Merz, N., Barnes, P. J., Brinton, R. D., Carrero, J. J., DeMeo, D. L., et al. (2020). Sex and Gender: Modifiers of Health, Disease, and Medicine. Lancet 396 (10250), 565-582. doi: 10.1016/S0140-6736(20)31561-0

Mbui, J., Olobo, J., Omollo, R., Solomos, A., Kip, A. E., Kirigi, G., et al. (2019). Pharmacokinetics, Safety, and Efficacy of an Allometric Miltefosine Regimen for the Treatment of Visceral Leishmaniasis in Eastern African Children: An Open-Label, Phase II Clinical Trial. Clin. Infect. Dis.: Off. Publ. Infect. Dis. Soc. America 68 (9), 1530-1538. doi: 10.1093/cid/ciy747

McSorley, H. J., and Maizels, R. M. (2012). Helminth Infections and Host Immune Regulation. Clin. Microbiol. Rev. 25 (4), 585-608. doi: 10.1128/CMR.05040-11

Miller, E., Warburg, A., Novikov, I., Hailu, A., Volf, P., Seblova, V., et al. (2014). Quantifying the Contribution of Hosts With Different Parasite Concentrations to the Transmission of Visceral Leishmaniasis in Ethiopia. PloS Negl. Trop. Dis. 8 (10), e3288. doi: 10.1371/journal.pntd.0003288

Mina, M. J., Kula, T., Leng, Y., Li, M., de Vries, R. D., Knip, M., et al. (2019). Measles Virus Infection Diminishes Preexisting Antibodies That Offer Protection From Other Pathogens. Science 366 (6465), 599-606. doi: $10.1126 /$ science.aay6485

Mishra, J., Madhubala, R., and Singh, S. (2013). Visceral and Post-Kala-Azar Dermal Leishmaniasis Isolates Show Significant Difference in Their In Vitro Drug Susceptibility Pattern. Parasitol. Res. 112 (3), 1001-1009. doi: 10.1007/ s00436-012-3222-1

Mitropoulos, K., Al Jaibeji, H., Forero, D. A., Laissue, P., Wonkam, A., LopezCorrea, C., et al. (2015). Success Stories in Genomic Medicine From ResourceLimited Countries. Hum. Genomics 9(1), 11. doi: 10.1186/s40246-015-0033-3

Mohapatra, T. M., Singh, D. P., Sen, M. R., Bharti, K., and Sundar, S. (2010). Compararative Evaluation of Rk9, Rk26 and Rk39 Antigens in the Serodiagnosis of Indian Visceral Leishmaniasis. J. Infect. Dev. Ctries. 4 (2), 114-117. doi: 10.3855/jidc.544

Molina, R., Ghosh, D., Carrillo, E., Monnerat, S., Bern, C., Mondal, D., et al. (2017). Infectivity of Post-Kala-Azar Dermal Leishmaniasis Patients to Sand Flies: Revisiting a Proof of Concept in the Context of the Kala-Azar Elimination Program in the Indian Subcontinent. Clin. Infect. Dis.: Off. Publ. Infect. Dis. Soc. America 65 (1), 150-153. doi: 10.1093/cid/cix245

Mondal, D., Bern, C., Ghosh, D., Rashid, M., Molina, R., Chowdhury, R., et al. (2019). Quantifying the Infectiousness of Post-Kala-Azar Dermal Leishmaniasis Towards Sandflies. Clin. Infect. Dis.: Off. Publ. Infect. Dis. Soc. America 69(2), 251-258. doi: 10.1093/cid/ciy891

Mondal, D., Ghosh, P., Khan, M. A., Hossain, F., Bohlken-Fascher, S., Matlashewski, G., et al. (2016). Mobile Suitcase Laboratory for Rapid Detection of Leishmania Donovani Using Recombinase Polymerase Amplification Assay. Parasit. Vectors 9 (1), 281. doi: 10.1186/s13071-016$1572-8$

Mondal, D., Kumar, A., Sharma, A., Ahmed, M. M., Hasnain, M. G., Alim, A., et al. (2019). Relationship Between Treatment Regimens for Visceral Leishmaniasis and Development of Post-Kala-Azar Dermal Leishmaniasis and Visceral Leishmaniasis Relapse: A Cohort Study From Bangladesh. PloS Negl. Trop. Dis. 13 (8), e0007653. doi: 10.1371/journal.pntd.0007653

Moulik, S., Chaudhuri, S. J., Sardar, B., Ghosh, M., Saha, B., Das, N. K., et al. (2018). Monitoring of Parasite Kinetics in Indian Post-Kala-Azar Dermal Leishmaniasis. Clin. Infect. Dis.: Off. Publ. Infect. Dis. Soc. America 66 (3), 404410. doi: $10.1093 / \mathrm{cid} / \mathrm{cix} 808$

Mukherjee, S., Sengupta, R., Mukhopadhyay, D., Braun, C., Mitra, S., Roy, S., et al. (2019). Impaired Activation of Lesional CD8(+) T-Cells Is Associated With Enhanced Expression of Programmed Death-1 in Indian Post Kala-Azar Dermal Leishmaniasis. Sci. Rep. 9 (1), 762. doi: 10.1038/s41598-018-37144-y

Mukhopadhyay, D., Das, N. K., De Sarkar, S., Manna, A., Ganguly, D. N., Barbhuiya, J. N., et al. (2012). Evaluation of Serological Markers to Monitor the Disease Status of Indian Post Kala-Azar Dermal Leishmaniasis. Trans. $R$. Soc. Trop. Med. Hyg. 106 (11), 668-676. doi: 10.1016/j.trstmh.2012.07.005

Musa, A., Khalil, E., Hailu, A., Olobo, J., Balasegaram, M., Omollo, R., et al. (2012). Sodium Stibogluconate (SSG) \& Paromomycin Combination Compared to SSG for Visceral Leishmaniasis in East Africa: A Randomised Controlled Trial. PloS Negl. Trop. Dis. 6 (6), e1674. doi: 10.1371/journal.pntd.0001674

Musa, A. M., Khalil, E. A., Mahgoub, F. A., Elgawi, S. H., Modabber, F., Elkadaru, A. E., et al. (2008). Immunochemotherapy of Persistent Post-Kala-Azar Dermal Leishmaniasis: A Novel Approach to Treatment. Trans. R. Soc. Trop. Med. Hyg. 102 (1), 58-63. doi: 10.1016/j.trstmh.2007.08.006

Musa, A. M., Khalil, E. A., Mahgoub, F. A., Hamad, S., Elkadaru, A. M., and El Hassan, A. M. (2005). Efficacy of Liposomal Amphotericin B (AmBisome) in the Treatment of Persistent Post-Kala-Azar Dermal Leishmaniasis (PKDL). Ann. Trop. Med. Parasitol. 99 (6), 563-569. doi: 10.1179/136485905X514127

Musa, A. M., Khalil, E. A., Raheem, M. A., Zijlstra, E. E., Ibrahim, M. E., Elhassan, I. M., et al. (2002). The Natural History of Sudanese Post-Kala-Azar Dermal Leishmaniasis: Clinical, Immunological and Prognostic Features. Ann. Trop. Med. Parasitol. 96 (8), 765-772. doi: 10.1179/000349802125002211

Musa, A. M., Younis, B., Fadlalla, A., Royce, C., Balasegaram, M., Wasunna, M., et al. (2010). Paromomycin for the Treatment of Visceral Leishmaniasis in Sudan: A Randomized, Open-Label, Dose-Finding Study. PloS Negl. Trop. Dis. 4 (10), e855. doi: 10.1371/journal.pntd.0000855 
Nandy, A., Addy, M., Maji, A. K., Guha, S. K., Banerjee, D., and Chaudhuri, D. (1998). Recurrence of Kala-Azar After PKDL: Role of Co-Factors. Trop. Med. Int. Health: TM IH 3 (1), 76-78. doi: 10.1046/j.1365-3156.1998.00176.x

Neu, K. E., Tang, Q., Wilson, P. C., and Khan, A. A. (2017). Single-Cell Genomics: Approaches and Utility in Immunology. Trends Immunol. 38 (2), 140-149. doi: 10.1016/j.it.2016.12.001

Nuel, G., and Garcia, A. (2021). A Timed Tally Counter for Microscopic Examination of Thick Blood Smears in Malaria Studies. Malaria J. 20 (1), 6. doi: 10.1186/s12936-020-03530-z

Okwor, I., and Uzonna, J. (2008). Persistent Parasites and Immunologic Memory in Cutaneous Leishmaniasis: Implications for Vaccine Designs and Vaccination Strategies. Immunol. Res. 41 (2), 123-136. doi: 10.1007/s12026008-8016-2

Ostyn, B., Gidwani, K., Khanal, B., Picado, A., Chappuis, F., Singh, S. P., et al. (2011). Incidence of Symptomatic and Asymptomatic Leishmania Donovani Infections in High-Endemic Foci in India and Nepal: A Prospective Study. PloS Negl. Trop. Dis. 5 (10), e1284. doi: 10.1371/journal. pntd.0001284

Ozdemir, V., Williams-Jones, B., Glatt, S. J., Tsuang, M. T., Lohr, J. B., and Reist, C. (2006). Shifting Emphasis From Pharmacogenomics to Theragnostics. Nat. Biotechnol. 24 (8), 942-946. doi: 10.1038/nbt0806-942

Palic, S., Bhairosing, P., Beijnen, J. H., and Dorlo, T. P. C. (2019). Systematic Review of Host-Mediated Activity of Miltefosine in Leishmaniasis Through Immunomodulation. Antimicrobial. Agents Chemother. 63 (7), e02507e02518. doi: 10.1128/AAC.02507-18

Ramesh, V., Singh, R., Avishek, K., Verma, A., Deep, D. K., Verma, S., et al. (2015). Decline in Clinical Efficacy of Oral Miltefosine in Treatment of Post Kala-Azar Dermal Leishmaniasis (PKDL) in India. PloS Negl. Trop. Dis. 9 (10), e0004093. doi: 10.1371/journal.pntd.0004093

Regional Strategic Framework for Elimination of VL From SEA Region (2005-2015) (New Delhi: WHO-SEARO. SEA-VBC-85), (Rev.1).

Rijal, S., Sundar, S., Mondal, D., Das, P., Alvar, J., and Boelaert, M. (2019). Eliminating Visceral Leishmaniasis in South Asia: The Road Ahead. BMJ 364, k5224. doi: 10.1136/bmj.k5224

Roden, D. M., McLeod, H. L., Relling, M. V., Williams, M. S., Mensah, G. A., Peterson, J. F., et al. (2019). Pharmacogenomics. Lancet 394 (10197), 521-532. doi: 10.1016/S0140-6736(19)31276-0

Rossi, M., Castiglioni, P., Hartley, M. A., Eren, R. O., Prevel, F., Desponds, C., et al. (2017). Type I Interferons Induced by Endogenous or Exogenous Viral Infections Promote Metastasis and Relapse of Leishmaniasis. Proc. Natl. Acad. Sci. U. S. A. 114 (19), 4987-4992. doi: 10.1073/pnas.1621447114

Rossi, M., and Fasel, N. (2018). How to Master the Host Immune System? Leishmania Parasites Have the Solutions! Int. Immunol. 30 (3), 103-111.

Saha, P., Ganguly, S., Chatterjee, M., Das, S. B., Kundu, P. K., Guha, S. K., et al. (2017). Asymptomatic Leishmaniasis in Kala-Azar Endemic Areas of Malda District, West Bengal, India. PloS Negl. Trop. Dis. 11 (2), e0005391. doi: 10.1371/journal.pntd.0005391

Salotra, P., Duncan, R. C., Singh, R., Subba Raju, B. V., Sreenivas, G., and Nakhasi, H. L. (2006). Upregulation of Surface Proteins in Leishmania Donovani Isolated From Patients of Post Kala-Azar Dermal Leishmaniasis. Microbes Infect. Inst. Pasteur 8 (3), 637-644. doi: 10.1016/j.micinf.2005.08.018

Salotra, P., Sreenivas, G., Pogue, G. P., Lee, N., Nakhasi, H. L., Ramesh, V., et al. (2001). Development of a Species-Specific PCR Assay for Detection of Leishmania Donovani in Clinical Samples From Patients With Kala-Azar and Post-Kala-Azar Dermal Leishmaniasis. J. Clin. Microbiol. 39 (3), 849-854. doi: 10.1128/JCM.39.3.849-854.2001

Samarasinghe, S. R., Samaranayake, N., Kariyawasam, U. L., Siriwardana, Y. D., Imamura, H., and Karunaweera, N. D. (2018). Genomic Insights Into Virulence Mechanisms of Leishmania Donovani: Evidence From an Atypical Strain. BMC Genomics 19 (1), 843. doi: 10.1186/s12864-018-5271-z

Schaefer, K. U., Kurtzhals, J. A., Kager, P. A., Gachihi, G. S., Gramiccia, M., Kagai, J. M., et al. (1994). Studies on the Prevalence of Leishmanin Skin Test Positivity in the Baringo District, Rift Valley, Kenya. Am. J. Trop. Med. Hyg. 50 (1), 7884. doi: 10.4269/ajtmh.1994.50.78

Singh, O. P., Gedda, M. R., Mudavath, S. L., Srivastava, O. N., and Sundar, S. (2019). Envisioning the Innovations in Nanomedicine to Combat Visceral Leishmaniasis: For Future Theranostic Application. Nanomed. (Lond.) 14 (14), 1911-1927. doi: 10.2217/nnm-2018-0448
Singh, O. P., Hasker, E., Boelaert, M., Sacks, D., and Sundar, S. (2020). Xenodiagnosis to Address Key Questions in Visceral Leishmaniasis Control and Elimination. PloS Negl. Trop. Dis. 14 (8), e0008363. doi: 10.1371/ journal.pntd.0008363

Singh, O. P., Syn, G., Nylen, S., Engwerda, C., Sacks, D., Wilson, M. E., et al. (2020). Anti-Interleukin-10 Unleashes Transcriptional Response to Leishmanial Antigens in Visceral Leishmaniasis Patients. J. Infect. Dis. 14 (8), e0008363. doi: 10.1093/infdis/jiaa381

Singh, O. P., Tiwary, P., Kushwaha, A. K., Singh, S. K., Singh, D. K., Lawyer, P., et al. (2021). Xenodiagnosis to Evaluate the Infectiousness of Humans to Sandflies in an Area Endemic for Visceral Leishmaniasis in Bihar, India: A Transmission-Dynamics Study. Lancet Microbe 2 (1), e23-e31. doi: 10.1016/ S2666-5247(20)30166-X

Snider, H., Lezama-Davila, C., Alexander, J., and Satoskar, A. R. (2009). Sex Hormones and Modulation of Immunity Against Leishmaniasis. Neuroimmunomodulation 16 (2), 106-113. doi: 10.1159/000180265

Sokal, J. E. (1975). Editorial: Measurement of Delayed Skin-Test Responses. New Engl. J. Med. 293 (10), 501-502. doi: 10.1056/NEJM197509042931013

Subba Raju, B. V., Singh, R., Sreenivas, G., Singh, S., and Salotra, P. (2008). Genetic Fingerprinting and Identification of Differentially Expressed Genes in Isolates of Leishmania Donovani From Indian Patients of Post-Kala-Azar Dermal Leishmaniasis. Parasitology 135 (Pt 1), 23-32. doi: 10.1017/ S0031182007003484

Sundar, S., and Singh, O. P. (2018). Molecular Diagnosis of Visceral Leishmaniasis. Mol. Diagn. Ther. 22 (4), 443-457. doi: 10.1007/s40291-018-0343-y

Sundar, S., Sinha, P. K., Rai, M., Verma, D. K., Nawin, K., Alam, S., et al. (2011). Comparison of Short-Course Multidrug Treatment With Standard Therapy for Visceral Leishmaniasis in India: An Open-Label, non-Inferiority, Randomised Controlled Trial. Lancet 377 (9764), 477-486. doi: 10.1016/S0140-6736(10) 62050-8

Tajebe, F., Getahun, M., Adem, E., Hailu, A., Lemma, M., Fikre, H., et al. (2017). Disease Severity in Patients With Visceral Leishmaniasis Is Not Altered by CoInfection With Intestinal Parasites. PloS Negl. Trop. Dis. 11 (7), e0005727. doi: 10.1371/journal.pntd.0005727

Tanak, A. S., Muthukumar, S., Krishnan, S., Schully, K. L., Clark, D. V., and Prasad, S. (2021). Multiplexed Cytokine Detection Using Electrochemical Point-of-Care Sensing Device Towards Rapid Sepsis Endotyping. Biosens. Bioelectron. 171, 112726. doi: 10.1016/j.bios.2020.112726

Tekola-Ayele, F., and Rotimi, C. N. (2015). Translational Genomics in Low- and Middle-Income Countries: Opportunities and Challenges. Public Health Genomics 18 (4), 242-247. doi: 10.1159/000433518

Telleria, E. L., Martins-da-Silva, A., Tempone, A. J., and Traub-Cseko, Y. M. (2018). Leishmania, Microbiota and Sand Fly Immunity. Parasitology 145 (10), 1336-1353. doi: 10.1017/S0031182018001014

Thakur, L., Kushwaha, H. R., Negi, A., Jain, A., and Jain, M. (2020). Leptomonas Seymouri Co-Infection in Cutaneous Leishmaniasis Cases Caused by Leishmania Donovani From Himachal Pradesh, India. Front. Cell. Infect. Microbiol. 10, 345. doi: 10.3389/fcimb.2020.00345

Topno, R. K., Madhukar, M., Pandey, K., Kumar, R., Rabidas, V. N., Kumar, M., et al. (2020). False Positivity of Rk39 Test in Five Chronic Myeloid Leukemia Cases From Bihar, India: A Possible Challenge to Leishmaniasis Diagnosis. Am. J. Trop. Med. Hyg. 103 (6), 2257-2259. doi: 10.4269/ajtmh.20-0301

van den Bogaart, E., Berkhout, M. M., Nour, A. B., Mens, P. F., Talha, A. B., Adams, E. R., et al. (2013). Concomitant Malaria Among Visceral Leishmaniasis in-Patients From Gedarif and Sennar States, Sudan: A Retrospective Case-Control Study. BMC Public Health 13, 332. doi: 10.1186/ 1471-2458-13-332

Van der Auwera, G., and Dujardin, J. C. (2015). Species Typing in Dermal Leishmaniasis. Clin. Microbiol. Rev. 28 (2), 265-294. doi: 10.1128/CMR. 00104-14

van Griensven, J., Carrillo, E., Lopez-Velez, R., Lynen, L., and Moreno, J. (2014). Leishmaniasis in Immunosuppressed Individuals. Clin. Microbiol. Infect.: Off. Publ. Eur. Soc. Clin. Microbiol. Infect. Dis. 20 (4), 286-299. doi: 10.1111/14690691.12556

van Griensven, J., Diro, E., Lopez-Velez, R., Ritmeijer, K., Boelaert, M., Zijlstra, E. E., et al. (2014). A Screen-and-Treat Strategy Targeting Visceral Leishmaniasis in HIV-Infected Individuals in Endemic East African Countries: The Way Forward? PloS Negl. Trop. Dis. 8 (8), e3011. doi: 10.1371/journal.pntd.0003011 
van Griensven, J., Mohammed, R., Ritmeijer, K., Burza, S., and Diro, E. (2018). Tuberculosis in Visceral Leishmaniasis-Human Immunodeficiency Virus Coinfection: An Evidence Gap in Improving Patient Outcomes? Open Forum Infect. Dis. 5 (4), ofy059. doi: 10.1093/ofid/ofy059

van Griensven, J., Zijlstra, E. E., and Hailu, A. (2014). Visceral Leishmaniasis and HIV Coinfection: Time for Concerted Action. PloS Negl. Trop. Dis. 8 (8), e3023. doi: 10.1371/journal.pntd.0003023

Verma, S., Avishek, K., Sharma, V., Negi, N. S., Ramesh, V., and Salotra, P. (2013). Application of Loop-Mediated Isothermal Amplification Assay for the Sensitive and Rapid Diagnosis of Visceral Leishmaniasis and Post-Kala-Azar Dermal Leishmaniasis. Diagn. Microbiol. Infect. Dis. 75 (4), 390-395. doi: 10.1016/j.diagmicrobio.2013.01.011

Verma, N., Bimal, S., Das, V. N., Pandey, K., Singh, D., Lal, C. S., et al. (2015). Clinicopathological and Immunological Changes in Indian Post Kala-Azar Dermal Leishmaniasis (PKDL) Cases in Relation to Treatment: A Retrospective Study. BioMed. Res. Int. 2015, 745062. doi: 10.1155/2015/745062

Verma, N., Singh, D., Pandey, K., Das, V. N., Lal, C. S., Verma, R. B., et al. (2013). Comparative Evaluation of PCR and Imprint Smear Microscopy Analyses of Skin Biopsy Specimens in Diagnosis of Macular, Papular, and Mixed PapuloNodular Lesions of Post-Kala-Azar Dermal Leishmaniasis. J. Clin. Microbiol. 51 (12), 4217-4219. doi: 10.1128/JCM.01482-13

Verrest, L., Kip, A. E., Musa, A., Schoone, G. J., Schallig, H., Mbui, J., et al. (2021). Blood Parasite Load as an Early Marker to Predict Treatment Response in Visceral Leishmaniasis in Eastern Africa. Clin. Infect. Dis.: Off. Publ. Infect. Dis. Soc. America 73(5), 775-782. doi: 10.1093/cid/ciab124

Vijayamahantesh,, Amit, A., Dikhit, M. R., Pandey, R. K., Singh, K., Mishra, R., et al. (2016). Elevated Serum ADA Activity as a Marker for Diagnosis and Prognosis of Visceral Leishmaniasis and Post Kala-Azar Dermal Leishmaniasis in Indian Patients. PloS One 11 (5), e0154117. doi: 10.1371/journal. pone. 0154117

Wasunna, M., Njenga, S., Balasegaram, M., Alexander, N., Omollo, R., Edwards, T., et al. (2016). Efficacy and Safety of AmBisome in Combination With Sodium Stibogluconate or Miltefosine and Miltefosine Monotherapy for African Visceral Leishmaniasis: Phase II Randomized Trial. PloS Negl. Trop. Dis. 10 (9), e0004880. doi: 10.1371/journal.pntd.0004880

WHO. (2011). Regional Strategic Framework for Elimination of Kala-Azar From the South-East Asia Region (2011-2015), SEA-CD-239. Available at: https:// apps.who.int/iris/handle/10665/205826.

WHO (2012) The Post-Kala-Azar Dermal Leishmaniasis (PKDL) Atlas: A Manual for Health Workers. Available at: https://appswhoint/iris/bitstream/handle/ $10665 / 1$.

WHO (2013) Post-Kala-Azar Dermal Leishmaniasis: A Manual for Case Management and Control: Report of a WHO Consultative Meeting, Kolkata, India, 2-3 July 2012. Available at: http://apps.who.int/iris/bitstream/10665/ 78608/1/9789241505215_eng.pdf (Accessed March 23, 2016).

WHO (2017) Self-Learning Online Course on Post-Kala-Azar Dermal Leishmaniasis (PKDL). Available at: https://extranetwhoint/elearn/course/ viewphp?id=29.

Williams, E., Isles, N. S., Seemann, T., Kilpatrick, T., Grigg, A., Leroi, M., et al. (2020). Case Report: Confirmation by Metagenomic Sequencing of Visceral Leishmaniasis in an Immunosuppressed Returned Traveler. Am. J. Trop. Med. Hyg. 103 (5), 1930-1933. doi: 10.4269/ajtmh.19-0841

World Health Organization = Organisation mondiale de la S (2020). Global Leishmaniasis Surveillance, 2017-2018, and First Report on 5 Additional Indicators - Surveillance Mondiale De La Leishmaniose, 2017-2018, Et Premier Rapport Sur 5 Indicateurs Supplémentaires. Wkly. Epidemiol. Rec. = Relevé Epidemiol. Hebd. 95 (25), 265-279.

Wu, Y., Tian, X., Song, N., Huang, M., Wu, Z., Li, S., et al. (2020). Application of Quantitative PCR in the Diagnosis and Evaluating Treatment Efficacy of Leishmaniasis. Front. Cell. Infect. Microbiol. 10, 581639. doi: 10.3389/ fcimb.2020.581639

Younis, B. M., Mohammed, H. A. A., Dafalla, M. M. M., Adam, A. O. A., Elamin, M. Y., Musa, A. M., et al. (2016). Cure of Post Kala-Azar Dermal Leishmaniasis With Paromomycin/Sodium Stibogluconate Combination: A Proof of
Concept. Int. J. Res. Med. Sci. 3 (1), 16-21 https://www.msjonline.org/index. php/ijrms/article/view/1200.

Younis, B. M., Osman, M., Khalil, E. A. G., Santoro, F., Furini, S., Wiggins, R., et al. (2021). Safety and Immunogenicity of ChAd63-KH Vaccine in Post-Kala-Azar Dermal Leishmaniasis Patients in Sudan. Mol. Ther.: J. Am. Soc. Gene Ther 29 (7), 2366-2377. doi: 10.1016/j.ymthe.2021.03.020

Zackay, A., Cotton, J. A., Sanders, M., Hailu, A., Nasereddin, A., Warburg, A., et al. (2018). Genome Wide Comparison of Ethiopian Leishmania Donovani Strains Reveals Differences Potentially Related to Parasite Survival. PloS Genet. 14 (1), e1007133. doi: 10.1371/journal.pgen.1007133

Zackay, A., Nasereddin, A., Takele, Y., Tadesse, D., Hailu, W., Hurissa, Z., et al. (2013). Polymorphism in the HASPB Repeat Region of East African Leishmania Donovani Strains. PloS Negl. Trop. Dis. 7 (1), e2031. doi: 10.1371/journal.pntd.0002031

Zijlstra, E. E. (2014). PKDL and Other Dermal Lesions in HIV Co-Infected Patients With Leishmaniasis: Review of Clinical Presentation in Relation to Immune Responses. PloS Negl. Trop. Dis. 8 (11), e3258. doi: 10.1371/ journal.pntd.0003258

Zijlstra, E. E. (2016). The Immunology of Post-Kala-Azar Dermal Leishmaniasis (PKDL). Parasit. Vectors 9, 464. doi: 10.1186/s13071-016-1721-0

Zijlstra, E. E. (2019). Biomarkers in Post-Kala-Azar Dermal Leishmaniasis. Front. Cell. Infect. Microbiol. 9, 228. doi: 10.3389/fcimb.2019.00228

Zijlstra, E. E., Daifalla, N. S., Kager, P. A., Khalil, E. A., El-Hassan, A. M., Reed, S. G., et al. (1998). Rk39 Enzyme-Linked Immunosorbent Assay for Diagnosis of Leishmania Donovani Infection. Clin. Diagn. Lab. Immunol. 5 (5), 717-720. doi: 10.1128/CDLI.5.5.717-720.1998

Zijlstra, E. E., and el-Hassan, A. M. (1993). Leishmanin and Tuberculin Sensitivity in Leishmaniasis in the Sudan, With Special Reference to Kala-Azar. Trans. $R$. Soc. Trop. Med. Hyg. 87 (4), 425-427. doi: 10.1016/0035-9203(93)90024-K

Zijlstra, E. E., and el-Hassan, A. M. (2001). Leishmaniasis in Sudan. Visceral Leishmaniasis. Trans. R. Soc. Trop. Med. Hyg. 95 Suppl 1, S27-S58. doi: 10.1016/s0035-9203(01)90218-4

Zijlstra, E. E., el-Hassan, A. M., Ismael, A., and Ghalib, H. W. (1994). Endemic Kala-Azar in Eastern Sudan: A Longitudinal Study on the Incidence of Clinical and Subclinical Infection and Post-Kala-Azar Dermal Leishmaniasis. Am. J. Trop. Med. Hyg. 51 (6), 826-836. doi: 10.4269/ajtmh.1994.51.826

Zijlstra, E. E., Kumar, A., Sharma, A., Rijal, S., Mondal, D., and Routray, S. (2020). Report of the Fifth Post-Kala-Azar Dermal Leishmaniasis Consortium Meeting, Colombo, Sri Lanka, 14-16 May 2018. Parasit. Vectors 13 (1), 159. doi: 10.1186/s13071-020-04011-7

Zijlstra, E. E., Liberton, N., Musa, A. M., Te Slaa, S., and Wolff, J. (2020). ThreeDimensional Optical Scanning in Post-Kala-Azar Dermal Leishmaniasis (PKDL). Am. J. Trop. Med. Hyg. 102 (2), 286-288. doi: 10.4269/ajtmh.19-0370

Zijlstra, E. E., Musa, A. M., Khalil, E. A., el-Hassan, I. M., and el-Hassan, A. M. (2003). Post-Kala-Azar Dermal Leishmaniasis. Lancet Infect. Dis. 3 (2), 87-98. doi: 10.1016/S1473-3099(03)00517-6

Conflict of Interest: The author declares that the research was conducted in the absence of any commercial or financial relationships that could be construed as a potential conflict of interest.

Publisher's Note: All claims expressed in this article are solely those of the authors and do not necessarily represent those of their affiliated organizations, or those of the publisher, the editors and the reviewers. Any product that may be evaluated in this article, or claim that may be made by its manufacturer, is not guaranteed or endorsed by the publisher.

Copyright (C) 2021 Zijlstra. This is an open-access article distributed under the terms of the Creative Commons Attribution License (CC BY). The use, distribution or reproduction in other forums is permitted, provided the original author(s) and the copyright owner(s) are credited and that the original publication in this journal is cited, in accordance with accepted academic practice. No use, distribution or reproduction is permitted which does not comply with these terms. 